Cita bibliográfica: Sánchez-Sánchez, F. J. y Sánchez-Sánchez ,A. M. (2021). Medición de la eficiencia laboral del turismo rural sostenible en espacios naturales protegidos de España. Investigaciones Turísticas (22), pp. 200-230. https://doi.org/10.14198/INTURI2021.22.9

\title{
Medición de la eficiencia laboral del turismo rural sostenible en espacios naturales protegidos de España
}

\section{Measurement of the labor efficiency of sustainable rural tourism in protected natural spaces in Spain}

Francisca J. Sánchez-Sánchez iD, Universidad Pablo de Olavide, España fsansan@upo.es

Ana M. Sánchez-Sánchez (iD, Universidad Pablo de Olavide , España amsansan@upo.es

\section{RESUMEN}

El turismo rural debe respetar y preservar el medio ambiente y servir de ayuda a la diversificación económica. El objetivo del estudio es evaluar la capacidad del turismo rural como instrumento para favorecer la eficiencia laboral y el desarrollo sostenible en espacios naturales protegidos. Se consideran factores laborales y de servicios de 55 zonas turísticas españolas emplazadas en espacios naturales protegidos, aplicándose técnicas de Análisis Envolvente de Datos y de Análisis Multivariante. La eficiencia del empleo en el turismo rural que se desarrolla en espacios naturales protegidos es poco significativa, siendo pocas las zonas turísticas que la alcanzan. El elevado proteccionismo de estas figuras de protección medioambiental, con planteamientos aislacionistas no favorece la eficiencia. El turismo rural puede ser un elemento de desarrollo sostenible del territorio, ayudando a la activación de recursos y al reequilibrio del territorio, pero para ello es importante conseguir la integración de los espacios naturales protegidos en las zonas rurales a través del planteamiento de modelos de gestión medioambiental adecuados.

Palabras clave: Turismo rural; Empleo; Espacios protegidos; Sostenibilidad.

\section{ABSTRACT}

Rural tourism must respect and preserve the environment and help economic diversification. The objective of this study is to evaluate the capacity of rural tourism as an instrument to promote labor efficiency and sustainable development in protected natural areas. Labor and service factors of 55 Spanish tourist areas located in protected natural areas are considered, applying techniques of Data Envelope Analysis and Multivariate Analysis. The efficiency of 
employment in rural tourism developed in protected natural areas is not very significant, with few tourist areas reaching some level of efficiency. The high protectionism of these environmental protection figures, with isolationist approaches, does not favor efficiency. Rural tourism can constitute an element of sustainable development of the territory, helping to activate resources and rebalance the territory. But for this, it is important to achieve the integration of protected natural spaces in rural areas by implementing management models suited to the environmental conditions.

Keywords: Rural tourism; Job; Protected spaces; Sustainability.

\section{INTRODUCCIÓN}

En el IV Congreso de Parques Nacionales y Áreas Protegidas, realizado en Caracas en 1992, se define espacio natural protegido como una zona de tierra y/o mar que se dedica a la protección y conservación de la diversidad biológica de los recursos naturales y culturales (Gómez-Limón et al. 2000). La Unión Mundial para la Conservación de la Naturaleza (UICN, 1994) define los espacios naturales protegidos como espacios geográficos claros que están determinados, reconocidos y gestionados mediante medios legales $u$ otros tipos de medios para lograr la preservación a largo plazo del medio natural, de los servicios eco sistémicos y de los valores culturales asociados. Deduciéndose de estas definiciones que estos espacios son imprescindibles para favorecer el mantenimiento, preservación y variedad de los espacios naturales, siendo también un medio excelente para el desarrollo sostenible de actividades económicas como el turismo.

En los últimos tiempos, el turismo que se desarrolla en zonas naturales protegidas, ha sufrido un considerable incremento. En el año 2007 la Organización Mundial del Turismo fijaba la tasa de crecimiento anual del turismo de naturaleza entre el $15 \%$ y el $20 \%$, desde entonces esta tasa no ha hecho más que crecer. En España, desde los años 70, el crecimiento de la afluencia turística a zonas protegidas ha sido muy notable, la primera estimación conocida consta del año 1973 donde se recoge un total de 4,2 millones de visitantes, en 1982 se tienen 20 millones de turistas y 25,5 millones en 1999 (Europarc, 2009). En el año 2016, el número de visitas realizadas en España a parques nacionales fue de 15,4 millones y de 15 millones de visitas a parques naturales (Europac, 2018). Este aumento tan considerable es debido a cambios culturales y motivacionales de los turistas (Corraliza et al., 2002; Eagles et al., 2002; Epler, 2002; Dearden et al., 2003; Boned et al., 2006). En España también ha contribuido a este incremento, el aumento considerable del número de espacios protegidos en los últimos años. En la actualidad, hay aproximadamente unos 900 espacios naturales protegidos (Figura 1), de los cuales 15 son parques nacionales, 152 parques naturales, 291 reservas naturales, 342 monumentos naturales, 57 paisajes protegidos y 2 áreas marinas protegidas (Europarc, 2018), suponiendo el $27 \%$ de la superficie terrestre protegida y el $13 \%$ de la superficie marina. 
Figura 1. Zonas naturales protegidas en España en 2018

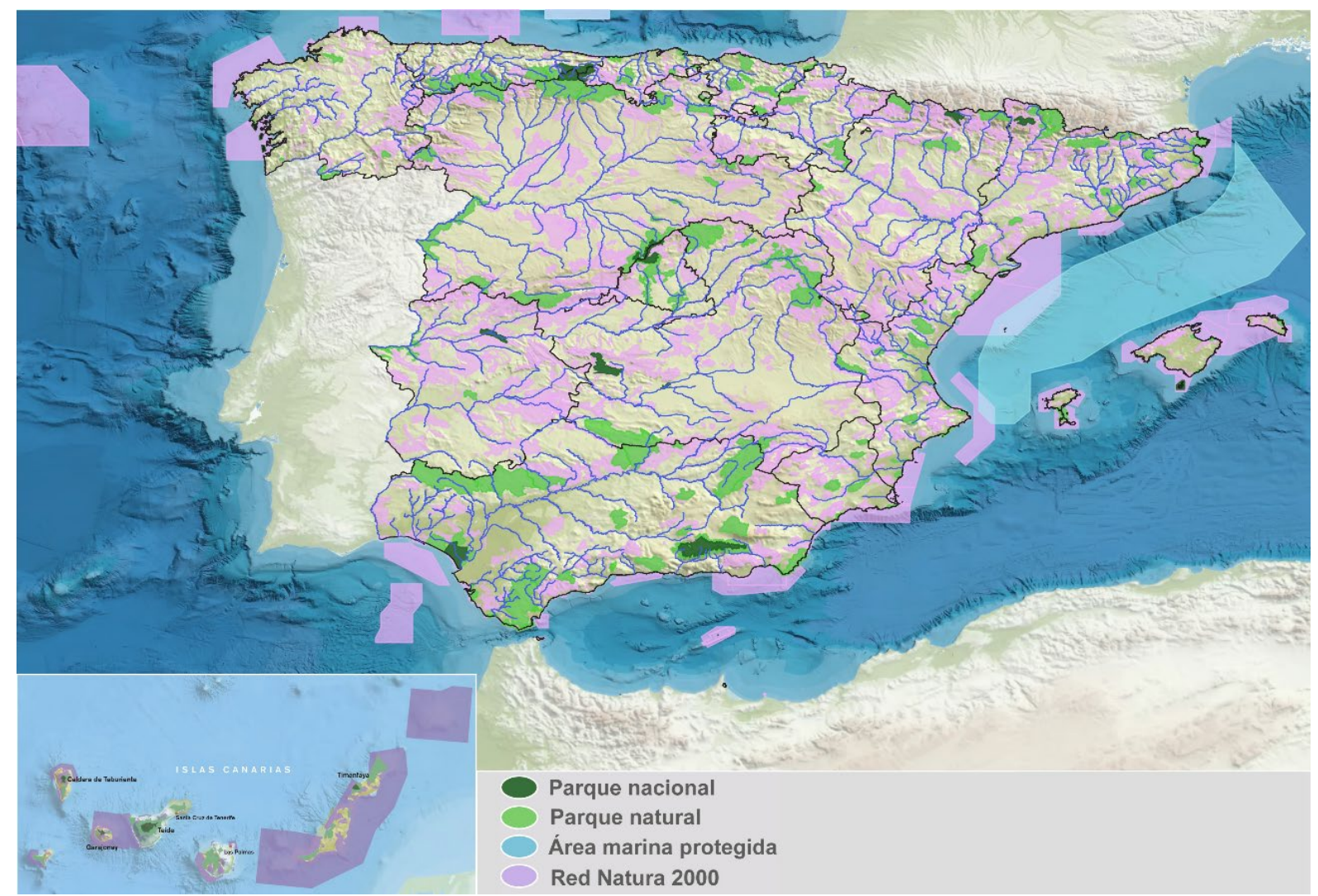

Fuente: Europarc España.

Las zonas naturales protegidas tienen cada vez mayor importancia territorial, esto requiere la evolución de estos espacios en instrumentos dinámicos de desarrollo sostenible, requiriendo el impulso de herramientas que favorezcan la protección medioambiental para ayudar a la relación entre sostenibilidad y turismo, lo que beneficiará al desarrollo local, económico, social y cultural del medio rural (Butler,1999, 2011; Sharpley y Roberts, 2004; Su, 2011 y Troitiño et al., 2005). La literatura muestra el vínculo entre conservación del medio ambiente en zonas naturales protegidas y el turismo en dichas zonas. Algunos de estos estudios demuestran el impacto negativo que puede tener el turismo mal gestionado en el medio ambiente (Green, Hunter y Moore, 1990; Hardy y Beeton, 2001; Huybers y Bennett, 2003), pero al mismo tiempo, puede ser un buen recurso medioambiental que proporciona un atractivo importante a la zona (Williams y Ponsford, 2009; Wearing y Neil, 2009). El turismo tiene un componente crítico que hay que tener en cuenta en la gestión de los espacios naturales protegidos (Eagles, McCool y Haynes, 2002). Dada la capacidad del turismo para provocar un impacto positivo, en aspectos de empleo, riqueza, dinamismo, mejora de ingresos, infraestructura, bienes, etc. (Egbali et al. 2011, Shariff y Abidin, 2013), el desafío es gestionar el turismo minimizando el impacto negativo de éste en el medio ambiente y la conservación de los ecosistemas locales (Blanke y Chiesa, 2008). Esto es especialmente importante en países como España, donde el turismo es un factor importante para el desarrollo. 
Las zonas rurales tienen un papel clave en el sector turístico, ayudan a preservar zonas ecológicas, la cultura tradicional y el patrimonio etnocultural (Stetic, 2012). De la Torre et al. (2005) destaca la importancia del turismo rural como un factor clave para el desarrollo socioeconómico, mientras que Giannakis (2014) remarca la importancia de las zonas rurales dentro de la Unión Europea desde el punto de vista del territorio que ocupan (91\%) y de la población que habita en ellas (59\%). Estas zonas rurales pueden revitalizarse mediante un turismo adecuado basado en el desarrollo sostenible (Garau, 2015). El turismo rural debe respetar y preservar el medio ambiente en el que se desarrolla, al tiempo que apoya la diversificación económica de manera viable a largo plazo (Puczkó y Rátz, 2000). La gestión de las zonas naturales protegidas es difícil dadas las múltiples normativas existentes que en ocasiones llegan a ser ambiguas (Naughton-Treves, Holland y Brandon, 2005). La cuestión clave es saber si las autoridades responsables tienen la capacidad de gestionar eficientemente dichas zonas y si dicha gestión se está aplicando en la práctica (Hockings et al., 2006). Es complicado definir modelos de desarrollo y ordenación territorial que equilibren la protección medioambiental y la promoción (Troitiño, 1995). Las administraciones tienen muchas dificultades para implantar políticas de conservación activa e impulsar programas de desarrollo sostenible que posibiliten superar la separación entre protección y desarrollo (Troitiño et al., 2005).

En España el turismo rural actúa como dinamizador de los espacios rurales, pero cabe plantearse si este efecto también es positivo y eficiente en espacios naturales protegidos. Tratar de dar respuesta a esta cuestión es la principal motivación del estudio. La hipótesis que fundamenta el trabajo es saber si la capacidad que tienen los espacios protegidos en España como instrumento de desarrollo sostenible es limitada, por ello es preciso determinar si estas zonas turísticas que gozan de especial protección medioambiental necesitan mejorar su desempeño para ser más eficientes. Se estudia si las figuras de protección medioambiental presentan mecanismos de protección demasiado restrictivos que provocan que el sector del turismo rural no alcance la eficiencia laboral. El objetivo fundamental de este trabajo es evaluar el impacto del turismo rural para actuar como herramienta de desarrollo sostenible en espacios naturales protegidos, analizándose el posible equilibrio entre el turismo rural como actividad económica sostenible y su potencial como herramienta para generar empleo y desarrollo de forma eficiente.

Para alcanzar el objetivo del estudio, se aplica la metodología utilizada habitualmente en estudios de medición de la eficiencia, basada en un método no paramétrico de programación matemática, conocido como Análisis Envolvente de Datos (DEA). La medición de la eficiencia es un tema de creciente interés, debido fundamentalmente al entorno tan competitivo en el que vivimos, en él se busca mejorar la rentabilidad y el uso óptimo y eficiente de los recursos (Tavares, 2002; Seiford, 1997). La definición de eficiencia establece: "the ability to avoid waste, either by producing as much output as technology and input usage allow or by using as little input as required by technology and production function" (Fried et al., 2008, p. 5). En otras palabras, la eficiencia está asociada con la máxima producción (output) que se puede alcanzar usando determinados recursos (inputs). En este trabajo se estudia la eficiencia laboral en el sector turístico, entendida esta como el uso mínimo de recursos turísticos (inputs) para alcanzar una producción o empleo (output) determinado. 
En los últimos años han sido muchos los trabajos que han aplicado la técnica DEA para analizar distintos aspectos de la eficiencia de la actividad turística, como por ejemplo, en estudios sobre hoteles (Ohe y Peypoch, 2016; Manasakis et al., 2013; Assaf et al., 2012; Ling-FengHsieh y HungLin, 2010; Perrigot et al., 2009), sobre agencias de viajes (ZhiYi, 2015; Fuentes, 2011; Köksala y Aksub, 2007; Barros y Dieke, 2007) o sobre aerolíneas (Semenick y Sickles, 2000; Adler y Golany, 2001; Scheraga, 2004; Barbot et al., 2008). También existen aplicaciones DEA en el sector del turismo rural, algunos de estos estudios analizan la productividad en segmentos específicos del turismo como en Pestana (2005), que toma como referencia las Pousadas de Portugal. Otros estudios distinguen entre eficiencia técnica y de escala (Pestana y Mascarenhas, 2005). Sin embargo, es difícil encontrar trabajos que apliquen esta metodología a espacios naturales protegidos debido a la dificultad de encontrar datos válidos. Entre los pocos trabajos relacionados con este tema se encuentran Hof et al. (2004), Bosetti y Locatelli (2006), Beech et al. (2008) y Valdivieso et al. (2015).

\section{TURISMO RURAL SOSTENIBLE: IMPACTO EN EL EMPLEO Y EL DESARROLLO LOCAL}

El turismo rural sostenible según la Countryside Commision (1995) consiste en "encontrar una armonía correcta en la relación que se establece entre las necesidades del visitante, el lugar y la comunidad receptora". Mientras que el desarrollo sostenible del turismo surge para garantizar un sector turístico eficiente basado en tres componentes principales: 1 . Intereses ambientales, que suponen el cumplimiento de las necesidades de la generación actual sin poner en peligro las necesidades de las generaciones futuras; 2 . Necesidades socioculturales, respeto por la cultura y medios de vida de la zona receptora sin imponer nuevos modelos y 3 . Económicas de las comunidades implicadas, suponiendo la contribución a los ingresos y a la creación de empleo de la población local (Mansfeld y Jonas, 2006). La Organización Mundial del Turismo (OMT, 2004) establece una relación de interdependencia entre crecimiento económico, conservación ambiental y distribución equitativa de beneficios, fijándolos como ejes fundamentales del desarrollo turístico sostenible.

El turismo rural se desarrolla de una forma local y sin grandes aglomeraciones turísticas, permitiendo establecer una correlación entre turismo sostenible y desarrollo sostenible (Sharpley, 2000, 2003). Habitualmente se asume que el turismo rural es intrínsecamente sostenible debido a que el número de turistas que atrae es reducido, no necesitando de una amplia red de infraestructuras. Los turistas muestran interés por la comunidad local visitada y sobre todo por el atractivo del paisaje y el ambiente en el que se realiza. Uno de los componentes principales del desarrollo rural sostenible es la comunidad local, se espera que esta comunidad esté plenamente concienciada con el desarrollo sostenible del entorno y tenga voluntad de apoyar el desarrollo turístico (Eshliki y Kaboudi, 2017; Huh y Vogt, 2008; Bestard y Nadal, 2007). El turismo sostenible está relacionado con las buenas prácticas y mejoras producidas en el entorno, especialmente en aspectos relacionados con el medio ambiente; sin embargo, es difícil implementarlo de forma práctica y adecuada (Mihalic, 2016). En España los modelos establecidos por las Comunidades Autónomas, así como el desarrollo producido en el turismo rural ponen en duda la intrínseca sostenibilidad del sector (Cánoves et al. 2004). 
Algunas razones de esto son el fraccionamiento de este turismo, la implantación de actividades no integradas en el entorno, la oferta turística que impone pocos mecanismos de control, lo que puede llevar al poco desarrollo de algunas zonas, a la saturación de otras y al deterioro de espacios ecológicos (Middleton y Hawkings, 1998).

La compleja interacción de la actividad turística en el entorno donde se desarrolla, da como resultado el conocido como impacto turístico (Mathieson y Wall, 1982), pudiendo tener un efecto positivo o negativo (Yoon et al., 1999). Este impacto se puede clasificar en tres aspectos: económico, ambiental y sociocultural (Fennell, 2007), siendo numerosos los trabajos que lo estudian en la comunidad local en la que se desarrolla (Muresan et al., 2016; Min et al., 2012; Ramseook-Munhurrun y Naidoo, 2011; Eraqi, 2007; McGehee y Andereck, 2004; Ko y Stewart, 2002; Brida et al. 1999). El resultado de la evaluación de los impactos del turismo depende en gran medida de dos características de la actividad que se está desarrollando: el número total de llegadas de turistas y el grado de concentración de turistas en ciertas épocas del año (Martin et al., 2017; Martin et al., 2018). Los efectos negativos del turismo incluyen: saturación de espacios e instalaciones públicas, interrupción del estilo de vida de los lugareños, un aumento en los precios de las propiedades, problemas de seguridad, daños ambientales, un aumento en producción de residuos y uso intensivo de recursos (Almeida et al., 2016; Ramseook-Munhurrun y Naidoo, 2011; Puczkó y Rátz, 2000). La intensidad y el origen de los impactos negativos dependen de diferentes factores que condicionan la sostenibilidad del turismo no solo desde una perspectiva ambiental, sino también desde una perspectiva social y económica (Roberts y Hall, 2001). A pesar de los efectos negativos del turismo, el desarrollo turístico y la sostenibilidad pueden coexistir siempre que exista una estrategia adecuada para proteger el medio ambiente (García y Díaz, 2014). El desarrollo de la actividad turística también genera efectos positivos como la creación de empleo (Sánchez y Sánchez, 2018; Long, 2011; Andereck y Nyaupane, 2011; McGehee y Andereck, 2004), la diversificación de la economía local, el mantenimiento de los servicios locales, el contacto externo con comunidades aisladas o la repoblación (Cánoves et al., 2004; Paniagua, 2002; Roberts y Hall, 2001 ), la mejora de la calidad de vida de los lugareños, la mejora de la imagen pública de la región, la preservación del patrimonio cultural e incluso el desarrollo de la red empresarial (Andereck et al., 2005). Otros efectos positivos son una mejor valoración del entorno natural, mejoras relacionadas con el transporte y las instalaciones, y la interacción cultural (Almeida et al., 2016), así como su capacidad de ser más respetuoso con el medio ambiente que otras formas alternativas de desarrollo (Ceballos-Lascurain, 1996; Master, 1998).

En las últimas décadas, el turismo rural ha crecido en numerosos países pasando de ser una actividad comercial secundaria a establecerse como un sector independiente (Busby y Rendle, 2000). En Europa, se ha producido una reestructuración de las zonas rurales (Schmied, 2005; Woods, 2005, 2011; OCDE, 2006), lo que ha supuesto que el empleo en actividades tradicionales, como por ejemplo la agricultura, haya disminuido. En muchas zonas rurales, la composición de la población ha cambiado debido al envejecimiento y la despoblación, lo que ha afectado a las distintas estructuras sociales, causando problemas estructurales en términos de mayor emigración, desempleo y malos resultados económicos. En muchos países europeos, 
el desarrollo supone que las zonas rurales queden rezagadas mientras que se produce la expansión de las zonas metropolitanas. Habitualmente el turismo se muestra como un medio para contrarrestar este desarrollo negativo, pues en algunos casos, se ha mostrado que los entornos rurales atraen a nuevos habitantes, turistas, empresarios y empresas, pudiendo considerarse como un recurso para aumentar las opciones de trabajo y residencia en zonas rurales (Findlay et al. 2000; Müller 2006). El turismo potencialmente puede atraer inmigración, puesto que ofrece empleos orientados al sector servicios y oportunidades para la puesta en marcha de nuevos negocios (Findlay et al., 2000; Paniagua, 2002; Lundmark, 2006; Lundmark, et al. 2014). Por esta razón, un incremento en la demanda de servicios rurales puede favorecer la diversificación del mercado laboral, contribuyendo esto a la reestructuración de la economía local, desde el sector primario y la manufactura hasta una economía más orientada a los servicios. Esta transformación se ha observado en zonas rurales de Europa, presentándose como una "nueva economía rural" (Hill, 2005; Halseth et al., 2010; Shucksmith et al., 2011; Copus y Hörnström, 2011).

Es innegable la creciente importancia del turismo en la estructura económica de muchos países. La globalización actual (Loncar, 2005), la internacionalización y la regionalización representan desafíos para los países actuales (Turek, 1999), lo que pone de relieve la importancia y la necesidad de comprender las tendencias a largo plazo en el turismo (Nanic et al., 2016) y su eficiencia. En España el turismo rural presenta un potencial importante, en algo menos de veinte años se ha multiplicado por tres el número de personas empleadas en dicho sector (Figura 2). Es una actividad en crecimiento, particularmente en áreas donde la agricultura no es especialmente competitiva en términos económicos (Cánoves et al., 2004). El análisis de la situación en las zonas rurales pone de manifiesto que el sector agrícola está sumido en una tendencia de pérdida de empleo, algunas posibles causas de esta tendencia son la disminución de explotaciones agrícolas, el abandono de las tareas agrícolas y también por el cambio de sector de actividad (Escudero, 1986), así como la cada vez mayor mecanización del sector. Además, el sector agrario ha sufrido diversas reformas en la política agraria, algunas de las cuales han provocado la disminución de presupuestos o la aplicación de criterios de protección al medioambiente, etc., provocando una disminución de la renta derivada de la explotación del sector agrario, así como, una pérdida importante de puestos de trabajo del sector. El turismo rural se plantea como una solución para esta problemática (Jiménez, 2000; Plaza y Araque, 2000; Sánchez y Sánchez, 2018), siendo esencial para el progreso de determinados territorios (Ghaderi y Henderson, 2012; Panyik et al., 2011; Pérez y Zizumbo, 2014), implicando la transferencia de riqueza lo que favorece el desarrollo y el crecimiento económico de las zonas de destino, beneficiando a los residentes y a las comunidades cercanas considerándose un motor clave para el crecimiento global y para la creación de empleo (Sánchez y Sánchez, 2018; Long, 2011; Andereck y Nyaupane, 2011; McCool, 2006). A pesar de estos estudios que destacan el impacto positivo del turismo sobre el empleo, esta incidencia no implica un alto grado de eficiencia turística (Sellers-Rubio y Casado-Díaz, 2018; Valdivieso et al., 2015). 
Figura 2. Personal empleado en turismo rural en España (personas)

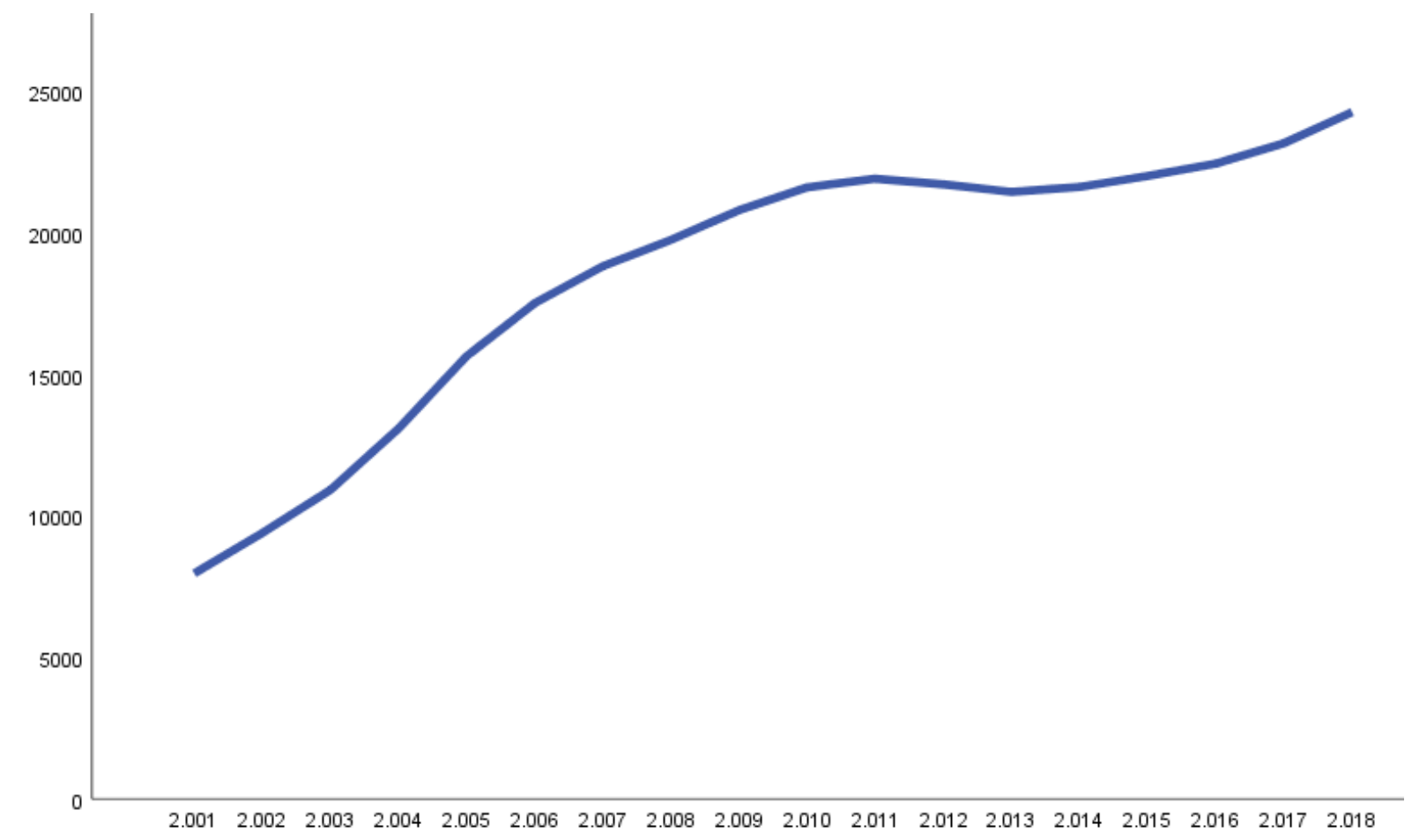

Fuente: Instituto Nacional de Estadística.

\section{DATOS Y METODOLOGÍA}

Los datos del estudio proceden de estadísticas oficiales publicadas por el Instituto Nacional de Estadística (INE). En concreto, los datos provienen de la Encuesta de Ocupación de Alojamientos de Turismo Rural del año 2017.

La unidad de análisis del estudio son las zonas turísticas definidas en España, por ello la selección de las variables usadas en la investigación están condicionadas a la disponibilidad de información de las bases de datos. El INE define zona turística como "aquella constituida por un conjunto de municipios en los que se localiza de forma específica la afluencia turística". Las diferentes zonas turísticas sobre las que se realiza el estudio, consideran áreas en las que destaca el turismo rural, algunas de las cuales están situadas en zonas costeras, en parques naturales y en parques nacionales. El análisis se centra en parques nacionales y naturales por ser la tipología de espacio protegido más relevante, siendo las figuras de protección de mayor rango. El estudio se realiza sobre 55 zonas turísticas que comprenden la totalidad del territorio español.

Para la selección de las variables se ha tenido en cuenta que el turismo se manifiesta básicamente en función del número de turistas, de sus necesidades de alojamiento, o de recursos en los que se basa el turismo como pueden ser las infraestructuras necesarias. La selección de inputs y outputs se basa en la revisión de la literatura y la disponibilidad de fuentes de datos fiables. En el trabajo de Sánchez y Sánchez (2018) se usan variables como el número de viajeros, de pernoctaciones, de establecimientos, de plazas ofertadas y personal empleado en turismo 
rural para analizar el impacto del turismo rural sobre el empleo, demostrándose la utilidad de dichas variables para caracterizar el factor que definen como Dinamismo Turístico-Laboral. Algunas de estas variables también son usadas frecuentemente en la literatura para estudiar la eficiencia turística (ver por ejemplo los trabajos de Deng, et al., 2019; Sellers-Rubio y CasadoDíaz, 2018; Soysal-Kurt, 2017; Hadad et al., 2012; Huang et al., 2012; Lozano y Gutiérrez, 2011; Hwang y Chang, 2003). Hockings et al. (2006) también usan algunas de estas variables para analizar la eficiencia en la gestión zonas naturales protegidas, estudiando en qué medida se protegen los valores de dichas zonas.

La Tabla 1 muestra las principales características de algunos trabajos relacionados con la eficiencia turística.

Tabla 1. Resumen de investigaciones empíricas en las que se aplica DEA en el sector turístico

\begin{tabular}{|c|c|c|c|c|c|}
\hline Investigación & Periodo & $\begin{array}{l}\text { Unidades } \\
\text { estudiadas }\end{array}$ & Inputs & Outputs & Modelo \\
\hline $\begin{array}{l}\text { Bosetti et al. } \\
(2003)\end{array}$ & $\begin{array}{l}\text { Datos } \\
\text { anuales } \\
\text { 2001-2002 }\end{array}$ & $\begin{array}{l}194 \\
\text { municipios } \\
\text { italianos }\end{array}$ & $\begin{array}{l}\text { Número de camas, } \\
\text { presión turística (turistas/ } \\
\text { superficie, turistas/ } \\
\text { habitantes locales), } \\
\text { distribución temporal } \\
\text { (turistas en julio-agosto/ } \\
\text { turistas por año) }\end{array}$ & $\begin{array}{l}\text { Tasa de uso (presencias } \\
\text { turísticas/camas),proxy de } \\
\text { beneficios ambientales }\end{array}$ & $\begin{array}{l}\text { BCC, CCR, } \\
\text { input y } \\
\text { output } \\
\text { orientados }\end{array}$ \\
\hline $\begin{array}{l}\text { Barros y } \\
\text { Mascarenhas } \\
(2005)\end{array}$ & $\begin{array}{l}\text { Datos } \\
\text { anuales } \\
\text { 1999-2001 }\end{array}$ & $\begin{array}{l}43 \text { Hoteles de } \\
\text { Portugal }\end{array}$ & $\begin{array}{l}\text { Número de empleados a } \\
\text { tiempo completo, valor en } \\
\text { libros de activos, número } \\
\text { de habitaciones }\end{array}$ & $\begin{array}{l}\text { Ventas, número de turistas, } \\
\text { número de noches pasadas }\end{array}$ & $\begin{array}{l}\text { Modelo de } \\
\text { asignación } \\
\text { DEA }\end{array}$ \\
\hline $\begin{array}{l}\text { Bosetti et al. } \\
(2006)\end{array}$ & $\begin{array}{l}\text { Datos } \\
\text { anuales } \\
\text { 2001-2003 }\end{array}$ & $\begin{array}{l}20 \text { regiones } \\
\text { italianas }\end{array}$ & $\begin{array}{l}\text { Tamaño del mercado, } \\
\text { índice de desarrollo } \\
\text { turístico, gasto público en } \\
\text { turismo, gasto público en } \\
\text { protección ambiental }\end{array}$ & $\begin{array}{l}\text { Número de turistas, } \\
\text { homogeneidad de los } \\
\text { flujos turísticos, índice de } \\
\text { eficiencia en el manejo } \\
\text { de residuos sólidos, } \\
\text { porcentaje de áreas } \\
\text { protegidas }\end{array}$ & CCR \\
\hline $\begin{array}{l}\text { Barros et al. } \\
\text { (2011) }\end{array}$ & $\begin{array}{l}\text { Datos } \\
\text { anuales } \\
\text { 2003-2007 } \\
\end{array}$ & $\begin{array}{l}22 \text { regiones } \\
\text { francesas }\end{array}$ & $\begin{array}{l}\text { Capacidad de alojamiento, } \\
\text { llegadas de turistas }\end{array}$ & Número de noches & $\begin{array}{l}\text { DEA en dos } \\
\text { etapas } \\
\text { con regresión }\end{array}$ \\
\hline $\begin{array}{l}\text { Barros et al. } \\
\text { (2011) }\end{array}$ & $\begin{array}{l}\text { Datos } \\
\text { anuales } \\
\text { 1998-2005 }\end{array}$ & $\begin{array}{l}15 \text { hoteles de } \\
\text { Portugal }\end{array}$ & $\begin{array}{l}\text { Número de trabajadores } \\
\text { a tiempo completo, valor } \\
\text { contable de la propiedad, } \\
\text { costos operativos. }\end{array}$ & Ventas, número de turistas & $\begin{array}{l}\text { DEA en dos } \\
\text { etapas } \\
\text { con regresión }\end{array}$ \\
\hline $\begin{array}{l}\text { Huang et al. } \\
\text { (2012) }\end{array}$ & $\begin{array}{l}\text { Datos } \\
\text { anuales } \\
\text { 2001-2006 }\end{array}$ & $\begin{array}{l}31 \text { regiones de } \\
\text { China }\end{array}$ & $\begin{array}{l}\text { Número de empleados a } \\
\text { tiempo completo, número } \\
\text { de turistas, activos fijos } \\
\text { totales }\end{array}$ & $\begin{array}{l}\text { Ingresos totales, tasa de } \\
\text { ocupación media }\end{array}$ & $\begin{array}{l}\text { DEA Window- } \\
\text { BCC }\end{array}$ \\
\hline $\begin{array}{l}\text { Hadad et al. } \\
\text { (2012) }\end{array}$ & 2008 & $\begin{array}{l}34 \text { países } \\
\text { desarrollados } \\
\text { y } 71 \text { en vías de } \\
\text { desarrollo }\end{array}$ & $\begin{array}{l}\text { Número de empleados, } \\
\text { número de habitaciones, } \\
\text { recursos naturales y } \\
\text { culturales }\end{array}$ & $\begin{array}{l}\text { Número de turistas, } \\
\text { gasto por turista }\end{array}$ & $\begin{array}{l}\text { Super- } \\
\text { eficiencia }\end{array}$ \\
\hline
\end{tabular}




\begin{tabular}{|c|c|c|c|c|c|}
\hline $\begin{array}{l}\text { Solana-Ibáñez et } \\
\text { al. (2016) }\end{array}$ & $\begin{array}{l}\text { Datos } \\
\text { anuales } \\
2005-2013\end{array}$ & $\begin{array}{l}17 \text { regiones de } \\
\text { España }\end{array}$ & $\begin{array}{l}\text { Número de camas } \\
\text { disponibles, número de } \\
\text { noches }\end{array}$ & $\begin{array}{l}\text { Número de personas que } \\
\text { se quedan al menos una } \\
\text { noche, determinantes de } \\
\text { eficiencia (atractivos y } \\
\text { servicios turísticos) destino } \\
\text { costero, número de bienes } \\
\text { culturales, número de } \\
\text { museos y colecciones, } \\
\text { porcentaje de asistencia } \\
\text { a reuniones, número de } \\
\text { clubes de golf federados, } \\
\text { número de restaurantes, } \\
\text { número de minoristas }\end{array}$ & $\begin{array}{l}\text { DEA (SBM), } \\
\text { indice de } \\
\text { productividad } \\
\text { Malmquist }\end{array}$ \\
\hline $\begin{array}{l}\text { Soysal-Kurt } \\
\text { (2017) }\end{array}$ & 2013 & $\begin{array}{l}29 \text { países } \\
\text { europeos }\end{array}$ & $\begin{array}{l}\text { Número de empleados en } \\
\text { el sector turístico, gastos } \\
\text { en turismo, número de } \\
\text { camas }\end{array}$ & $\begin{array}{l}\text { Llegadas de turistas, } \\
\text { ingresos por turismo, } \\
\text { número de pernoctaciones }\end{array}$ & $\begin{array}{l}\text { CCR, input } \\
\text { orientado }\end{array}$ \\
\hline $\begin{array}{l}\text { Cvetkosa y } \\
\text { Barišić (2017) }\end{array}$ & $\begin{array}{l}\text { Datos } \\
\text { anuales } \\
2010-2015 \\
\end{array}$ & $\begin{array}{l}11 \text { países } \\
\text { balcánicos }\end{array}$ & $\begin{array}{l}\text { Exportaciones de } \\
\text { visitantes, viajes } \\
\text { nacionales, gasto turístico }\end{array}$ & $\begin{array}{l}\text { Contribución total de viajes } \\
\text { y turismo al PIB, } \\
\text { empleo }\end{array}$ & $\begin{array}{l}\text { BCC, output } \\
\text { orientado, } \\
\text { DEA Window }\end{array}$ \\
\hline $\begin{array}{l}\text { Sellers-Rubio } \\
\text { y Casado-Díaz } \\
(2018)\end{array}$ & $\begin{array}{l}\text { Datos } \\
\text { anuales } \\
\text { 2008-2016 }\end{array}$ & & $\begin{array}{l}\text { Número de hoteles, } \\
\text { número de camas, número } \\
\text { de empleados, duración } \\
\text { de estancia, número de } \\
\text { turistas internacionales } \\
\text { que llegan a España, } \\
\text { producto turístico de sol y } \\
\text { playa, número de hoteles } \\
\text { distinguidos con distinción } \\
\text { de calidad }\end{array}$ & $\begin{array}{l}\text { Gasto medio diario, } \\
\text { ingresos por habitación, } \\
\text { tasa media de ocupación }\end{array}$ & DEA \\
\hline
\end{tabular}

Nota: CCR indica el modelo de Cooper-Charnes-Rhodes, BCC indica el modelo de Banker-Charnes-Cooper y SBM indica Slacks-Based Measure.

Elaboración propia.

La matriz de datos está formada por 5 variables recogidas para las 55 zonas turísticas españolas.

Para el análisis envolvente de datos (DEA) se considerará un output, personal empleado en turismo rural $\left(y_{1}\right)$, y cuatro inputs: Viajeros $\left(x_{1}\right)$, pernoctaciones $\left(x_{2}\right)$, establecimientos abiertos $\left(x_{3}\right)$ y plazas ofertadas $\left(x_{4}\right)$. La descripción de las variables consideradas junto con algunas medidas estadísticas asociadas, se recogen en la Tabla 2. 
Tabla 2. Descripción de los datos

\begin{tabular}{|c|c|c|c|c|}
\hline Variables & Descripción & Unidades & Media & $\begin{array}{l}\text { Desviación } \\
\text { típica }\end{array}$ \\
\hline $\mathrm{y}_{1}$ & $\begin{array}{l}\text { Personal: empleados que trabajan en turismo } \\
\text { rural en zonas turísticas }\end{array}$ & Personas & 202.56 & 263.456 \\
\hline $\mathrm{x}_{1}$ & Viajeros: personas que hacen un viaje turístico & Personas & $34,473.78$ & $44,844.780$ \\
\hline$x_{2}$ & $\begin{array}{l}\text { Pernoctaciones: cada noche que un viajero se } \\
\text { aloja en un establecimiento }\end{array}$ & Personas & $107,642.33$ & $150,590.251$ \\
\hline$x_{3}$ & $\begin{array}{l}\text { Establecimientos: lugar en el que se realiza } \\
\text { una actividad de turismo rural }\end{array}$ & Número & 182.16 & 303.699 \\
\hline$x_{4}$ & $\begin{array}{l}\text { Plazas: total de camas con las que cuenta un } \\
\text { establecimiento de alojamiento turístico }\end{array}$ & Número & $1,554.11$ & $2,226.660$ \\
\hline
\end{tabular}

Elaboración propia.

Para comprobar que los inputs y output seleccionados son adecuados, se realiza una prueba de isotonicidad (Sigala et al., 2004; Chiang, 2006). Esta prueba se fundamenta en la hipótesis de que los inputs y output deben estar correlacionados positivamente, así cuanto mayor sea el valor de los inputs, mayor será el output. La Tabla 3 muestra los coeficientes de correlación de Pearson por pares entre los cuatro inputs y el output, así como los p-valores ( $p$ ) para testar la significatividad de la correlación entre cada par de variables estudiadas.

Tabla 3. Coeficientes de correlación de Pearson y p-valores

\begin{tabular}{llllll}
\hline & Viajeros & Pernoctaciones & Establecimientos & Plazas & Personal \\
\hline Viajeros & 1 & $0,965^{* *}$ & $0,354^{* *}$ & $0,554^{* *}$ & $0,947^{* *}$ \\
& & $(0,000)$ & $(0,008)$ & $(0,000)$ & $(0,000)$ \\
Pernoctaciones & $0,965^{* *}$ & 1 & $0,382^{* *}$ & $0,577^{* *}$ & $0,973^{* *}$ \\
& $(0,000)$ & & $(0,004)$ & $(0,000)$ & $(0,000)$ \\
Establecimientos & $0,354^{* *}$ & $0,382^{* *}$ & 1 & $0,962^{* *}$ & $0,410^{* *}$ \\
& $(0,008)$ & $(0,004)$ & & $(0,000)$ & $(0,002)$ \\
Plazas & $0,554^{* *}$ & $0,577^{* *}$ & $0,962^{* *}$ & 1 & $0,582^{* *}$ \\
& $(0,000)$ & $(0,000)$ & $(0,000)$ & & $(0,000)$ \\
Personal & $0,947^{* *}$ & $0,973^{* *}$ & $0,410^{* *}$ & $0,582^{* *}$ & 1 \\
& $(0,000)$ & $(0,000)$ & $(0,002)$ & $(0,000)$ & \\
\hline
\end{tabular}

$* p<0,05 * * p<0,01$. Elaboración propia.

Los inputs han sido correctamente elegidos, dado que son estadísticamente significativos, y además tienen correlación positiva con el output. Todos los inputs están asociados positivamente entre sí, lo que muestra que las zonas turísticas con más turistas, también poseen más pernoctaciones, más establecimientos turísticos y ofertan más plazas en turismo rural. Estas asociaciones positivas reflejan una posible redundancia en los factores de los inputs 
y output seleccionados, lo que conllevará intentar reducir la dimensión de los datos mediante la aplicación de técnicas de Análisis Multivariante.

El análisis descriptivo de los datos, permite mostrar para cada zona turística, el personal empleado en el sector (Figura 3), destacando en este aspecto, las Baleares (Illes): Isla de Mallorca, Pirineus, Pirineo Aragonés y Pirineo Navarro. El análisis que se aplicará, permitirá comprobar si estas zonas importantes en cuanto a personal empleado, utilizan eficientemente los recursos de los que disponen.

Figura 3. Número de personas empleadas en turismo rural según zona turística.

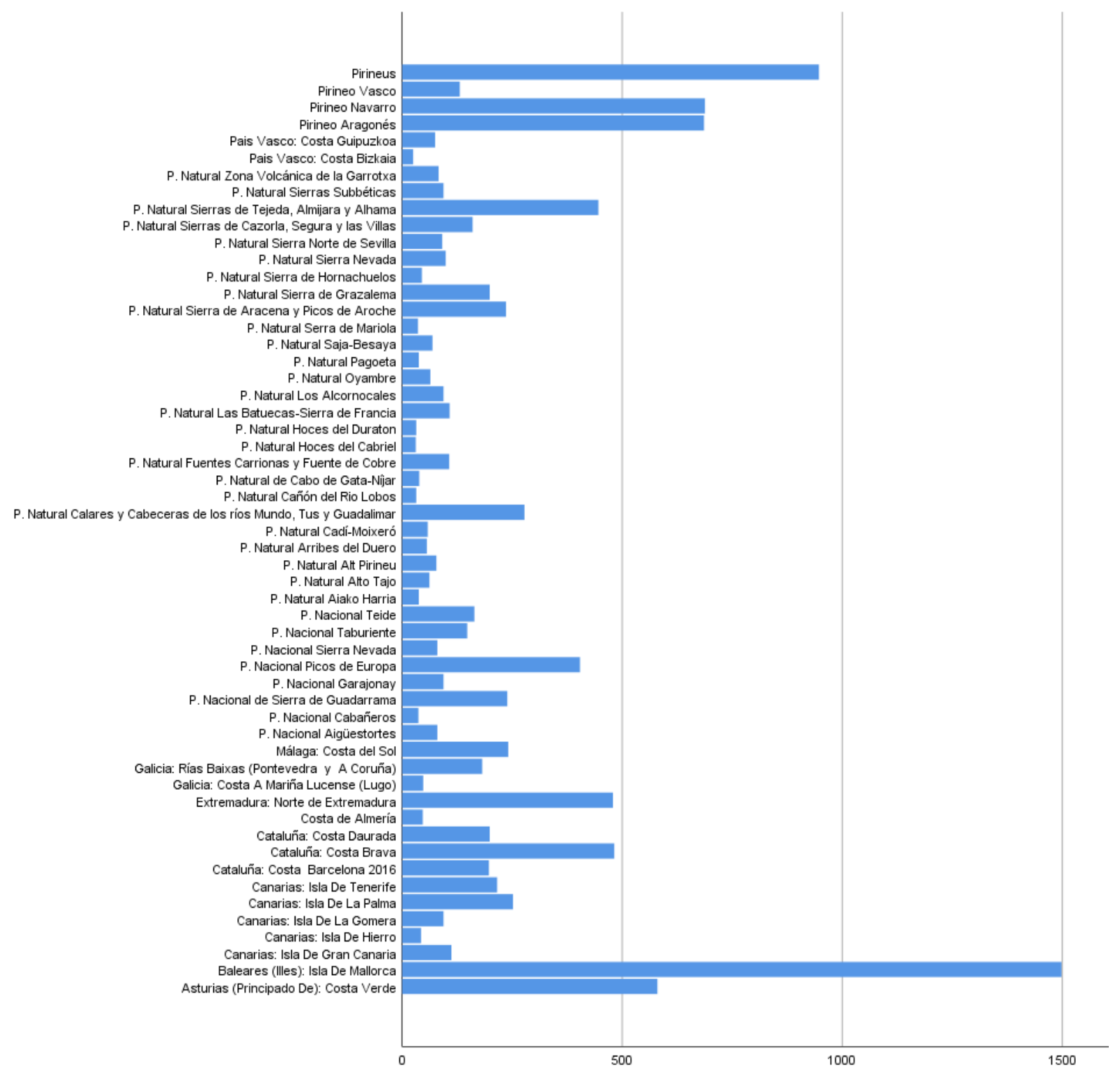

Elaboración propia. 


\subsection{Análisis Envolvente de Datos (DEA)}

El análisis envolvente de datos (DEA) es una técnica no paramétrica usada para comparar la eficiencia en la actividad de un conjunto de organizaciones (DMU) en base a los datos que contienen información sobre ciertas variables. Las variables se clasifican como inputs $u$ outputs de acuerdo al proceso de producción, esto es, los inputs son los recursos que influyen en la producción de los outputs.

EI DEA asigna un valor de eficiencia a cada DMU para comparar entre DMUs eficientes y DMUs ineficientes. Si el valor de eficiencia para un determinado DMU es igual a 1 entonces dicha DMU se dice que es completamente eficiente, mientras que si una DMU obtiene un valor de eficiencia por debajo de 1 entonces se dice que dicha DMU es ineficiente.

En el estudio se usa el modelo estándar CCR DEA orientado al input introducido por Charnes et al., 1978. Sea $U=\{1,2, \ldots, u\}$ un conjunto de DMUs independientes, donde cada uno consume un conjunto de diferentes inputs, $\mathrm{I}=\{1,2, \ldots, \mathrm{n}\}$, en cuantías $x_{i j}$ para crear un conjunto de distintos outputs, $\mathrm{O}=\{1,2, \ldots, \mathrm{m}\}$, en cuantías $y_{k j}$. El valor de eficiencia de un DMU, $j_{0} \in U$, específico se calcula de la siguiente manera:

$$
\begin{aligned}
& E\left(j_{0}\right)=\min \theta_{j_{0}} \\
& \text { s.a. } \quad \sum_{j \in U} \lambda_{j} x_{i j} \leq \theta_{j_{0}} x_{i j_{0}} \quad \text { paratodo } i \in I \\
& \sum_{j \in U} \lambda_{j} y_{k j} \geq y_{k j_{0}} \quad \text { paratodo } k \in O \\
& \lambda_{j} \geq 0 \quad \text { paratodo } j \in U \\
& \theta_{j_{0}} \quad \text { libre. }
\end{aligned}
$$

Según los valores de eficiencia obtenidos del modelo antes indicado, se podría definir un ranking de DMUs ineficientes, pero las DMUs eficientes no se pueden ordenar en estos términos ya que todas éstas tienen el mismo valor de eficiencia (todas las eficientes valen 1 ). Hay diferentes enfoques en DEA que desarrollan los modelos básicos para fijar un ranking adicional de DMUs eficientes y no sólo de DMUs ineficientes. Dos de los métodos más usados son el Global Leader (Oral y Yolalan, 1990) y el de la puntuación de super-eficiencia (Andersen y Petersen, 1993). El término Global Leader, destaca la unidad que presenta mejor rendimiento global. Se clasifica como Global Leader, la unidad eficiente que aparece con mayor frecuencia en los conjuntos de referencia de las unidades ineficientes. El método de la puntución super-eficiente excluye del conjunto inicial de DMUs a la DMU a la que se está calculando el valor de eficiencia. Este método puede alcanzar valores más grandes que 1, pudiéndose usar para ordenar todas las DMUS. El método de super-eficiencia tiene la ventaja respecto a otros, de que se aplica para ordenar sólo las DMUs eficientes, dado que los valores de super-eficiencia de las DMUs ineficientes son los mismos que sus valores de eficiencia. Este será el método seleccionado para establecer el ranking de unidades eficientes. 
El valor de super-eficiencia de cada DMU, , para el modelo estándar CCR DEA orientado al input, se obtiene de la siguiente forma:

$$
\begin{gathered}
E^{\text {super }}\left(j_{0}\right)=\min \theta_{j_{0}} \\
\text { s.a. } \\
\sum_{\substack{j \in U \backslash\left\{j_{0}\right\} \\
\lambda_{j} \geq 0}} \lambda_{j} x_{i j} \leq \theta_{j_{0}} x_{i j_{0}} \quad \text { paratodo } i \in y_{k j_{0}} \quad \text { paratodo } k \in O \\
\theta_{j_{0}} \text { libre. }
\end{gathered}
$$

Algunas de las principales ventajas de la metodología DEA son: 1) Permite trabajar con inputs y outputs con diferentes unidades. 2) No necesita suponer el pleno empleo de los factores productivos. 3) No requiere el uso de formas funcionales explícitas. 4) Las DMUs se comparan con una DMU ideal construida a partir del desempeño de DMU "pares" reales, productivamente más eficientes, mediante el cálculo de una combinación lineal de estas últimas. Por otro lado, algunas desventajas de esta metodología son: 1) Es sensible a los errores de medición. 2) La omisión de variables puede provocar ineficiencias. 3) No estima bien eficiencias (o ineficiencias) "absolutas", donde el objetivo sea obtener resultados potenciales o ideales. 4) Al tratarse de una técnica no paramétrica, complica la formulación de test de hipótesis estadísticos.

\subsection{Análisis Multivariante}

Como técnicas de Análisis Multivariante para el tratamiento de los datos, se aplicarán el Análisis Factorial y el Análisis Clúster.

\section{Análisis Factorial}

El Análisis Factorial persigue identificar las dimensiones que expliquen las correlaciones entre las variables, resumiendo la información original, mediante combinaciones de las variables. Se proporciona un número más pequeño de variables no observadas (dimensiones), explicando el comportamiento de sujetos en los que se han medido las variables originales.

El modelo factorial que se utiliza es lineal, donde se relacionan variables y dimensiones. Las variables observadas se expresan como composición lineal de dimensiones no observadas:

$$
X_{i j}=\sum_{k=1}^{m} \lambda_{i k} F_{k j}+U_{i j},
$$

donde $X_{i j}$ es el valor de la variable $X_{i}$ en la j-ésima observación (en nuestro caso en la zona turística j); $F_{k j}$ es el valor de la k-ésima dimensión en la zona turística $j$; $\lambda_{i k}$ es el coeficiente de regresión estandarizado de la variable $X_{i}$ sobre la dimensión común $F_{k}$ y $U_{i j}$ representa la 
parte del valor de la variable $X_{i}$ en la j-ésima zona que no está explicada por las dimensiones comunes del modelo.

Se utilizará el método de extracción de Componentes Principales (Morrison, 1987), para la obtención de dimensiones, pues el análisis que se aplica es exploratorio y no inferencial. Para la selección de dimensiones se usa el criterio de extraer aquellas cuyo autovalor sea superior a uno (Kaiser, 1960).

\section{Análisis Clúster}

Una vez extraídas las dimensiones mediante el análisis factorial, estas se utilizarán para clasificar a las zonas turísticas en grupos con características análogas mediante el Análisis Clúster. Así, el Análisis Clúster persigue identificar casos similares basándose en las características que poseen éstos, de forma que los casos que pertenecen a un mismo clúster o grupo son lo más parecidos posible entre sí y además son, lo más desiguales posible entre los distintos grupos (Hair et al. (2000)). Como medida de similitud entre los sujetos (en nuestro estudio las zonas turísticas), se tomará la distancia euclídea al cuadrado, aplicándose el método no jerárquico de k-medias.

\section{RESULTADOS}

El análisis de la eficiencia laboral para las zonas turísticas españolas se presenta distinguiendo dos casos: En primer lugar, se presenta el ranking de zonas eficientes en el empleo del sector, para lo cual se aplicará DEA y en segundo lugar, se plantea el análisis de la eficiencia de las zonas turísticas, mediante la aplicación de técnicas de Análisis Multivariante.

\subsection{Ranking de eficiencia laboral por zonas turísticas}

Para la obtención de las zonas turísticas eficientes laboralmente es preciso la aplicación de DEA, lo que conlleva la resolución de 55 problemas de programación lineal. La Tabla 4 recoge el ranking de zonas turísticas eficientes laboralmente, mostrándose la puntuación de eficiencia en dichas zonas en sentido decreciente. Un total de 8 zonas turísticas alcanzan la eficiencia laboral, lo que permite indicar que, a pesar del amplio desarrollo del turismo rural en España, aún falta para que este tenga un efecto eficiente e importante sobre el empleo de las zonas en las que se desarrolla. Es interesante que 3 de las 8 zonas turísticas en las que se alcanza la eficiencia laboral son zonas costeras, por lo que se puede sospechar que en los resultados obtenidos no solo influye el turismo rural, posiblemente también tenga una importancia destacada el turismo de costa. También destaca que entre las zonas eficientes no hay ningún parque nacional español, solo alcanzan la eficiencia dos parques naturales, quizás los parques nacionales y naturales son figuras de protección demasiado restrictivas para favorecer la eficiencia en el empleo. 
Tabla 4. Ranking y puntuaciones de eficiencia por provincias.

\begin{tabular}{|c|c|c|c|c|c|}
\hline & Zona turística & $\begin{array}{l}\text { Puntuación } \\
\text { eficiencia }\end{array}$ & & Zona turística & $\begin{array}{l}\text { Puntuación } \\
\text { eficiencia }\end{array}$ \\
\hline 1 & Baleares (Illes): Isla De Mallorca & 2,9214090 & 29 & P. Nacional Picos de Europa & 0,6753297 \\
\hline 2 & $\begin{array}{l}\text { P. Natural Calares y Cabeceras de los ríos } \\
\text { Mundo, Tus y Guadalimar }\end{array}$ & 1,5886550 & 30 & $\begin{array}{l}\text { P. Natural Fuentes Carrionas y Fuente de } \\
\text { Cobre }\end{array}$ & 0,6643913 \\
\hline 3 & Isla De Hierro (Canarias) & 1,5139180 & 31 & P. Nacional Teide & 0,6496693 \\
\hline 4 & P. Natural Hoces del Cabriel & 1,3150580 & 32 & $\begin{array}{l}\text { Galicia: Rías Baixas (Pontevedra y A } \\
\text { Coruña) }\end{array}$ & 0,6428746 \\
\hline 5 & $\begin{array}{l}\text { P. Natural Sierra de Aracena y Picos de } \\
\text { Aroche }\end{array}$ & 1,2274910 & 33 & P. Nacional Sierra Nevada & 0,6382862 \\
\hline 6 & Isla De La Palma (Canarias) & 1,1297960 & 34 & P. Natural Sierra Nevada & 0,6316687 \\
\hline 7 & Pirineus & 1,1033350 & 35 & Isla De Tenerife (Canarias) & 0,6236606 \\
\hline 8 & Pirineo Aragonés & 1,0225400 & 36 & P. Natural Sierra de Hornachuelos & 0,6231212 \\
\hline 9 & P. Natural Cadí-Moixeró & 0,9190261 & 37 & P. Natural Oyambre & 0,6208338 \\
\hline 10 & Pirineo Navarro & 0,8927717 & 38 & Costa del Sol (Málaga) & 0,6097961 \\
\hline 11 & P. Nacional Taburiente & 0,8918059 & 39 & P. Natural Sierras Subbéticas & 0,6044576 \\
\hline 12 & $\begin{array}{l}\text { P. Natural Sierras de Tejeda, Almijara y } \\
\text { Alhama }\end{array}$ & 0,8686958 & 40 & P. Nacional Aigüestortes & 0,6012014 \\
\hline 13 & Norte de Extremadura & 0,8410321 & 41 & P. Natural Arribes del Duero & 0,597365 \\
\hline 14 & P. Natural Sierra de Grazalema & 0,8249224 & 42 & Costa A Mariña Lucense (Galicia) & 0,5482633 \\
\hline 15 & $\begin{array}{l}\text { P. Natural Sierras de Cazorla, Segura y las } \\
\text { Villas }\end{array}$ & 0,8136393 & 43 & P. Natural Saja-Besaya & 0,5407662 \\
\hline 16 & Isla De La Gomera (Canarias) & 0,8110370 & 44 & P. Natural Cañón del Rio Lobos & 0,5395117 \\
\hline 17 & P. Nacional Garajonay & 0,8110370 & 45 & P. Natural Hoces del Duraton & 0,5243611 \\
\hline 18 & Costa Daurada (Cataluña) & 0,7914141 & 46 & P. Natural Alt Pirineu & 0,5205891 \\
\hline 19 & Isla De Gran Canaria (Canarias) & 0,7893632 & 47 & P. Natural Aiako Harria & 0,4606068 \\
\hline 20 & P. Natural Las Batuecas-Sierra de Francia & 0,7845247 & 48 & P. Nacional Cabañeros & 0,4360132 \\
\hline 21 & P. Natural Los Alcornocales & 0,7640258 & 49 & P. Natural Zona Volcánica de la Garrotxa & 0,4226661 \\
\hline 22 & Costa Verde (Asturias) & 0,7633177 & 50 & Pirineo Vasco & 0,4120366 \\
\hline 23 & P. Nacional de Sierra de Guadarrama & 0,7404403 & 51 & Costa Bizkaia (País Vasco) & 0,3833661 \\
\hline 24 & P. Natural Sierra Norte de Sevilla & 0,7399979 & 52 & P. Natural Pagoeta & 0,3768139 \\
\hline 25 & P. Natural Serra de Mariola & 0,7035290 & 53 & Costa Guipuzkoa (País Vasco) & 0,3662908 \\
\hline 26 & Costa Brava (Cataluña) & 0,6943849 & 54 & P. Natural de Cabo de Gata-Níjar & 0,3342197 \\
\hline 27 & P. Natural Alto Tajo & 0,6911184 & 55 & Costa de Almería & 0,2829236 \\
\hline 28 & Costa Barcelona (Cataluña) & 0,6830989 & & & \\
\hline
\end{tabular}

Elaboración propia.

Algunas de las zonas turísticas eficientes laboralmente destacan claramente en cuanto al número de personas empleadas (ver Figura 3 y Tabla 4), como es el caso de Baleares (Illes), Pirineus yPirineo Aragonés, sin embargo, otras zonas eficientes como Isla de Hierro, P. Natural Calares y Cabeceras de los ríos Mundo, tus y Guadalimar, P. Natural Hoces del Cabriel, P. Natural Hoces del Cabriel, P. Natural Sierra de Aracena y Picos de Aroche e Isla de la Palma no tienen valores destacados en cuanto a personal empleado, sin embargo, alcanzan la eficiencia, evidenciando que en estas zonas se utilizan eficientemente los recursos de los que disponen. Otro aspecto interesante es que el Pirineo Navarro a pesar de destacar claramente en cuanto a personal empleado en el sector (Figura 3), no logra alcanzar la eficiencia laboral (Tabla 4).

En cuanto al análisis de la puntuación de eficiencia, la puntuación media de eficiencia laboral de las zonas turísticas españolas es de 0,7631 puntos, comprobándose que algo más de 
un tercio de estas zonas ( 22 de 55) alcanzan una puntuación de eficiencia por encima del valor medio, confirmándose los malos resultados en cuanto a utilización óptima de recursos de las zonas turísticas españolas en cuanto a maximización del empleo.

\subsection{Análisis Multivariante}

Para la segunda parte del análisis se aplica un Análisis Factorial y un Análisis Clúster, con el objetivo de determinar las posibles relaciones entre las zonas turísticas utilizando los inputs y output antes estudiados, junto con la puntuación de eficiencia obtenida.

\section{Análisis Factorial}

Para comprobar si el modelo factorial es adecuado, se contrasta si la matriz de correlaciones de las variables es una matriz identidad. Para ello, se usa la prueba de esfericidad de Bartlett, donde el estadístico de Bartlett alcanza el valor de 575,937, de forma que cuanto mayor sea dicho estadístico, y por tanto menor el nivel de significación $(0,0000)$, menos posible resulta que la matriz de correlaciones sea la matriz identidad y con ello, más apropiado es emplear el Análisis Factorial, que es lo que sucede en nuestro caso.

Se seleccionan dos dimensiones que explican el 90,853\% de la variabilidad total (ver Tabla 5). Siendo un porcentaje de varianza explicada bastante bueno (Hair et al., 2000 fijan una cota inferior del $60 \%$ para aceptar el modelo en estudios relacionados con Ciencias Sociales).

Tabla 5. Factores extraídos y varianza explicada

\begin{tabular}{lccc}
\hline & Autovalores & \% de varianza & \% varianza acumulada \\
\hline Dimensión 1. Eficiencia laboral-demanda turística & 4,043 & 67,376 & 67,376 \\
Dimensión 2. Duración de la estancia & 1,409 & 23,477 & 90,853 \\
\hline
\end{tabular}

Elaboración propia.

En la Tabla 6 se muestra la matriz factorial rotada, indicando los coeficientes de correlación lineal entre las dimensiones y las variables. Cada valor recoge el peso de cada variable en cada dimensión, de modo que las dimensiones con pesos altos en términos absolutos indican una relación importante con las variables.

Tabla 6. Pesos de las variables en las dimensiones

\begin{tabular}{lc|lc}
\hline \multicolumn{1}{c|}{ Variables } & Dimensión 1 & \multicolumn{1}{c}{ Variables } & Dimensión 2 \\
\hline Personal empleado & $\mathbf{0 , 9 3 1}$ & Establecimientos & $\mathbf{0 , 9 7 8}$ \\
Pernoctaciones & $\mathbf{0 , 9 1 6}$ & Plazas & $\mathbf{0 , 9 4 8}$ \\
Viajeros & $\mathbf{0 , 8 8 2}$ & Personal empleado & 0,336 \\
Puntuación de eficiencia & $\mathbf{0 , 8 1 8}$ & Pernoctaciones & 0,335 \\
Plazas & $\mathbf{0 , 2 9 6}$ & Viajeros & 0,332 \\
Establecimientos & 0,094 & Puntuación de eficiencia & $-0,096$ \\
\hline
\end{tabular}

Elaboración propia. 
La primera dimensión explica el 67,376\% de la variabilidad (Tabla 5), estando estrechamente relacionado con 4 de las 6 variables utilizadas (personal empleado, pernoctaciones, viajeros y puntuación de eficiencia). Esta relación está indicada por las correlaciones positivas y con valor mayor a 0,8 de las variables antes mencionadas (ver Tabla 5). Valores elevados (o bajos) de la dimensión están relacionados con zonas turísticas con elevados (o bajos) valores de personal empleado, de pernoctaciones, de viajeros y de puntuación de eficiencia. Por ello, a la primera dimensión, se le etiqueta como eficiencia laboral-demanda turística.

Las zonas turísticas que obtienen mayor puntuación en la primera dimensión, es decir, las más destacadas en cuanto a eficiencia laboral-demanda turística son por orden de relevancia, Baleares (Illes): Isla de Mallorca, con 5,67 puntos, Pirineus con 1,98 puntos, Pirineo Navarro con 1,47 puntos y el Pirineo Aragonés con 1,12 puntos.

La segunda dimensión explica el 23,477\% de la varianza total (Tabla 5). Las zonas turísticas que componen dicha dimensión presentan una relación directa y estrecha con las variables número de establecimientos y de plazas (Tabla 5). Valores elevados (o bajos) de la dimensión están asociados con zonas turísticas con elevados (o bajos) valores en cuanto a establecimientos y plazas ofertadas. Por ello, se etiqueta a la segunda dimensión como recursos turísticos.

Las zonas turísticas que obtienen mejor puntuación en la segunda dimensión son: Costa del Sol (Málaga) con 6,01 puntos, Pirineus con 1,90 puntos, Costa Verde (Asturias) con 1,25 puntos y el Pirineo Aragonés con 1,21 puntos. Obsérvese que no destaca ninguna zona turística en ninguna de las dimensiones que esté situada en parque nacional o natural. También destaca que las zonas más importantes tanto en la primera como en la segunda dimensión son zonas costeras, lo que parece poner de manifiesto que existe una coexistencia positiva y complementaria entre el turismo rural y de litoral.

\section{Análisis Clúster}

El objetivo que se persigue con el análisis clúster es crear grupos de zonas turísticas con características similares en las dimensiones que se extrajeron anteriormente.

El método que se usará para determinar los clústers es el no jerárquico. Se aplicará en las puntuaciones obtenidas en las dos dimensiones extraídas según las zonas turísticas, necesitándose conocer con anterioridad el número de clúster. Por ello, en primer lugar, se aplica un análisis clúster mediante procedimientos jerárquicos, lo que permitirá conocer el número adecuado de clúster, junto con la distribución inicial para el método no jerárquico.

Se realizan distintos intentos, y por las diferentes variaciones que se producen en la varianza residual, se seleccionan 4 clúster como número óptimo. 
Tabla 7. Puntuaciones medias en los clústers

\begin{tabular}{lcccc}
\hline & \multicolumn{4}{c}{ Clúster } \\
\hline & $\mathbf{1}$ & $\mathbf{2}$ & $\mathbf{3}$ & $\mathbf{4}$ \\
\hline Dimensión 1. Eficiencia laboral-demanda turística & $-0,28443$ & $-1,63568$ & 1,09543 & 5,67169 \\
\hline Dimensión 2. Recursos turísticos & $-0,30029$ & 6,01178 & 0,98566 & $-0,38419$ \\
\hline
\end{tabular}

Elaboración propia.

La Tabla 8 recoge las zonas turísticas que componen cada uno de los clústers.

Tabla 8. Configuración de zonas turísticas en los clústers

\begin{tabular}{ll}
\hline Clúster & \multicolumn{1}{c}{ Zonas turísticas } \\
\hline 1 & 45 zonas. Cataluña: Costa de Barcelona, Costa Daurada; Costa de Almería; Canarias: Isla de Gran \\
& Canaria, Isla de la Palma, Isla de la Gomera, Isla del Hierro, Isla de Tenerife; Galicia: Rías Baixas, Costa \\
& A Mariña Lucense; País Vasco: Costa de Guipuzkoa, Costa de Bizkaia, Pirineo Vasco; Parque Nacional: \\
& Sierra Nevada, Cabañeros, Aigüestortes, Garajonay, Taburiente, Teide, Sierra de Guadarrama; \\
& Parque Natural: Alto Tajo, Cabo de Gata-Níjar, Los Alcornocales, Sierra de Grazalema, Sierra de \\
& Hornachuelos, Sierras Subbéticas, Sierra Nevada, Sierra de Aracena y Picos de Aroche, Sierras de \\
& Cazorla, Segura y las Villas, Sierra Norte de Sevilla, Oyambre, Fuentes Carrionas y Fuente de Cobre, \\
& Las Batuecas-Sierra de Francia, Arribes del Duero, Cañón del Río Lobos, Calares y Cabeceras de los \\
& Ríos Mundo, Tus y Guadalimar, Cadí-Moixeró, Alt Pirineu, Serra de Mariola, Hoces de Cabriel, Saja- \\
& Besaya, Hoces del Duratón, Zona Volcánica de la Garrotxa, Aiako Harria, Pagoeta. \\
& 1 zona. Costa del Sol (Málaga) \\
& 8 zonas. Costa Verde (Asturias), Costa Brava (Cataluña), Extremadura Norte, Pirineo Aragonés, \\
& Pirineus, Pirineo Navarro, Parque Nacional Picos de Europa, Parque Natural Sierras de Tejeda, \\
& Almijara y Alhama \\
& 1 zona. Baleares (Illes): Isla de Mallorca
\end{tabular}

Elaboración propia.

Según las puntuaciones medias de los clústers en los distintos factores (Tabla 7), se puede hacer una clasificación de las zonas turísticas en tres grupos: Zonas que tienen una eficiencia laboral y demanda turística buena, siendo el clúster 4 el que registra la puntuación media más elevada respecto a la primera dimensión. En concreto, las Baleares (Illes) en la Isla de Mallorca es la zona turística más destacada en cuanto a dicha dimensión. Las zonas que presentan una eficiencia laboral y demanda turística media son la Costa Verde (Asturias), Costa Brava (Cataluña), Extremadura Norte, Pirineo Aragonés, Pirineus, Pirineo Navarro, Parque Nacional Picos de Europa, Parque Natural Sierras de Tejeda, Almijara y Alhama), siendo zonas que obtienen la segunda puntuación media más elevada en la primera dimensión; y finalmente zonas turística que tienen una puntuación inferior a la media en el factor de eficiencia laboral y demanda turística (Tabla 7) las cuales presentan una eficiencia laboral mala, perteneciendo a él las zonas integrantes del clúster 1 y 2 . Obsérvese que la incidencia del empleo en el turismo rural que se desarrolla en zonas con espacios protegidos (parques nacionales y naturales) es poco relevante.

El clúster 2 registra la mayor puntuación media respecto a la segunda dimensión, indicando que la única zona turística que lo integra, la Costa del Sol, destaca especialmente en recursos dedicados al turismo. Las zonas turísticas que presentan la segunda puntuación media 
más elevada en la segunda dimensión, son la que integran el clúster 3: Costa Verde (Asturias), Costa Brava (Cataluña), Extremadura Norte, Pirineo Aragonés, Pirineus, Pirineo Navarro, Parque Nacional Picos de Europa, Parque Natural Sierras de Tejeda, Almijara y Alhama. Por el contrario, las zonas que pertenecen a los clústers 1 y 4 presentan una puntuación por debajo de la media en cuanto a recursos turísticos.

El análisis realizado, confirma que Baleares (Illes) es la única zona turística que alcanza la eficiencia laboral por cualquiera de las dos metodologías aplicadas (DEA y Análisis Multivariante), aunque también destacan en el aspecto laboral las zonas del Pirineo Aragonés y Pirineus. Obsérvese, que ninguna de las zonas catalogadas como parque nacional o natural destacada en cuanto eficiencia laboral. Estos resultados hacen pensar que el excesivo proteccionismo que las instituciones realizan en dichas zonas está limitando en gran medida el impacto sobre el empleo provocado por el turismo rural. Hay que encontrar un equilibrio sostenible entre la necesaria conservación de los espacios protegidos y el desarrollo de actividades que se realizan en dichas zonas, como el turismo, pues dicha relación puede influir al necesario desarrollo de determinadas zonas.

\section{DISCUSIÓN}

Sánchez-Zamora et al. (2014) muestran que el turismo rural es una actividad global en el territorio español, y además es una de las alternativas más relevante para la preparación de estrategias públicas que favorezcan el desarrollo de las zonas rurales. Nuestros resultados manifiestan que el sector turístico rural tiene una capacidad desigual para generar empleo de forma eficiente según la zona geográfica. Aunque el turismo rural permite reducir el desempleo en algunas zonas rurales, son pocas las zonas turísticas situadas en algún espacio con especial protección medioambiental que llegan a alcanzar la eficiencia laboral. Lo que, en cierta forma, refleja el fracaso relativo de los espacios protegidos españoles como instrumento de desarrollo sostenible (Mulero y Garzón, 2005; Troiño et al., 2005). Muchas de estas zonas turísticas necesitan mejorar su desempeño para ser más eficientes (Valdivieso et al., 2015).

Como en Muresan et al. (2016), nuestro estudio muestra que el factor ambiental es muy importante para el desarrollo territorial, además el alto potencial turístico natural y la conciencia de conservación natural de los espacios protegidos permiten ayudar al desarrollo sostenible. El desarrollo turístico y la sostenibilidad pueden coexistir, pero para ello debe existir una estrategia apropiada que permita la protección medioambiental (García y Díaz, 2014), evitando aislar los espacios naturales protegidos. Esta estrategia debe favorecer una gestión eficaz y equitativa (Woodley et al., 2012), buscando la harmonía entre la satisfacción de los turistas y las posibles amenazas internas a su integridad biofísica o cultural (Eagles y McCool, 2002). Es imprescindible el equilibrio entre el crecimiento del sector del turismo rural y la conservación del entorno. Por ello, es importante considerar el factor ambiental en los procesos de planificación turística (De Aranzábal et al., 2009), es necesaria la intervención sobre la transformación de los parajes para controlar y conservar las características diferenciales de cada zona y así evitar la degradación del paisaje. Ahmad et al. (2015) y Butler (2011) muestran que un turismo sostenible en zonas rurales favorece el desarrollo de éstas, haciendo necesario la implantación de estrategias que certifiquen la conservación ambiental y ecológica. Santos 
et al. (2016) establecen la necesidad de incorporar el paisaje como un factor más de competitividad en la planificación turística de los territorios, permitiendo adoptar políticas que fijen la conservación y mejora del paisaje, teniendo en cuenta la incidencia que el turismo tiene en los recursos socioculturales y ambientales de los lugares de destino (Deery et al., 2012) y su importancia para la gestión turística. Nuestros resultados muestran que, para realizar dicha planificación estratégica, debe tenerse en cuenta que una excesiva protección de determinadas zonas puede provocar el aislamiento de éstas, así como que el impacto del turismo en aspectos laborales y de desarrollo del territorio no sea positivo. Por tanto, debe realizarse una conservación de la naturaleza que sea compatible con el uso racional de recursos.

\section{CONCLUSIONES}

En las últimas décadas, las zonas rurales en España han sufrido una transformación intensa tanto en aspectos sociales, como económicos y culturales. La creciente importancia del sector del turismo rural dentro de la economía española, motiva el análisis de la eficiencia de las zonas rurales situadas en espacios naturales protegidos como destinos turísticos. El desarrollo económico en las zonas rurales en España sobre todo se apoya en el sector primario, aunque en los últimos años se han incorporado otras actividades, entre ellas el turismo, lo que favorece que los habitantes de dichas zonas dispongan de rentas adicionales. El turismo rural es un dinamizador significativo de los espacios rurales, pudiendo ser una importante herramienta para el desarrollo rural. Es necesario conseguir la estabilidad del sector para poder garantizar una alternativa sólida para el crecimiento económico, la creación de empleo y la utilización racional de recursos procedentes de zonas rurales.

Se toman como referencia para el estudio, las unidades territoriales definidas por zonas naturales turísticas que gozan de protección medioambiental, de forma que cada destino turístico se define por las características del entorno rural en el que se encuentra con factores de condicionamiento específicos.

Los resultados indican que el impacto del turismo sobre la eficiencia laboral en espacios naturales protegidos es poco relevante, no llegando a alcanzar la eficiencia laboral ninguna de las zonas turísticas situadas en parques nacionales o naturales. Solamente las Baleares (Illes) alcanza la eficiencia laboral, aunque también destacan en aspectos laborales las zonas del Pirineo Aragonés y Pirineus, pero sin llegar a la eficiencia. Las Baleares (Illes) es un destino turístico enfocado tradicionalmente al turismo de costa, el hecho de que sea la única zona turística que alcanza la eficiencia laboral pone de manifiesto que el flujo turístico desde el litoral a zonas rurales próximas, con significativos recursos naturales, ambientales y paisajísticos ayuda a dinamizar dichas zonas. Estas zonas ofrecen un turismo diferente al habitual, ofertando enclaves únicos, así como importantes recursos naturales y ambientales, permitiendo la integración del turismo rural y el de litoral, fortaleciéndose de esta forma ambos destinos turísticos, haciéndolos más competitivos y favoreciendo las características y sostenibilidad de los espacios naturales. El turismo rural y de litoral pueden ser complementarios, pudiendo coexistir favorablemente. 
Son pocas las zonas turísticas situadas en espacios naturales protegidos que destacan en cuanto a recursos turísticos ofertados, entendiendo estos como servicios de infraestructuras turísticas. Estos resultados muestran que en España los espacios naturales protegidos no resultan un instrumento especialmente útil de desarrollo sostenible. Esto puede ser debido a que las instituciones realizan una protección excesiva en zonas naturales protegidas, lo que está coartando en buena medida el impacto eficiente sobre el empleo del turismo rural. Se deben dejar atrás ideas proteccionistas demasiado restrictivas que logran aislar excesivamente a las zonas rurales, es necesario encontrar el equilibrio entre el crecimiento del sector turístico y la conservación del entorno. Además, la integración de la población local tanto en la gestión como en la conservación de dichos espacios sería un aspecto positivo a considerar, consiguiéndose de esta forma herramientas que impulsan el desarrollo y la sostenibilidad.

La falta de coordinación entre las administraciones responsables de la gestión de las áreas protegidas, de la actividad turística y del desarrollo rural, hace necesario que las administraciones realicen un esfuerzo para conseguir la integración de los espacios naturales protegidos en el marco de estrategias de cooperación y desarrollo territorial. Es necesaria una estrategia de ordenación e intervención en los espacios protegidos, de forma que sean definidos como una realidad turística en sí misma, siendo preciso establecer las bases tanto de la intervención pública como de las iniciativas privadas.

La utilización racional de los recursos de las zonas rurales y el planteamiento de modelos adecuados que permitan la gestión medioambiental, favorecerán la integración de los espacios naturales protegidos en su entorno. El desarrollo de nuevos enfoques debe intentar conseguir el reequilibrio territorial para que el turismo actúe como factor de progreso territorial y social. Los espacios naturales protegidos situados en áreas con situaciones económicas y sociales difíciles pueden ayudar a impulsar sus recursos, beneficiando con ello la innovación y el desarrollo del territorio.

Se debe apostar por un turismo respetuoso con la naturaleza y el entorno, lo que sin duda ayudará positivamente al turismo y en consecuencia favorecerá la eficiencia. También es importante el análisis del sector turístico desde la perspectiva del suministro para garantizar su sostenibilidad, dada la importancia que tienen los recursos naturales en sus múltiples figuras de protección, siendo necesario combinar los objetivos económicos que persigue el turismo rural con principios ecológicos y de desarrollo sostenible. Es importante tener un punto de vista estratégico del sector que agregue transversalmente la agricultura, el desarrollo y el turismo, sin que ello produzca una saturación del entorno rural, que logre ambientes sostenibles, generando empleo y riqueza. También hay que proporcionar instrumentos de desarrollo integrado en las zonas rurales, con políticas multidireccionales que ayuden a la población local a seguir viviendo en su residencia de origen, aumentando la inversión en educación, en infraestructuras, en sanidad y en asistencia, proporcionando ayudas para la innovación y para la creación de empresas. Invertir en el reequilibrio territorial de las zonas rurales es asignar sostenibilidad futura tanto a estos espacios naturales como a los residentes.

Para investigaciones futuras podría realizarse un análisis del sector, que estudie las particularidades de la demanda turística en las zonas rurales situadas en espacios naturales protegidos, con el propósito de estudiar la sostenibilidad del turismo en dichas zonas o incluso 
su incidencia sobre la población local. También se podría extender el estudio a otros países europeos donde la demanda de turismo rural sea semejante a la del territorio español, considerándose nuevas variables, como pueden ser aquellas que recojan el impacto ambiental en zonas protegidas.

En estudios futuros, además de analizar la eficiencia en la gestión o administración de las zonas protegidas se pueden comparar diferentes modelos de gestión y estudiar si existen diferencias entre ellos en cuanto a la eficiencia de desempeño en dichas zonas. Sin embargo, esto es especialmente difícil debido a la ausencia de datos que contengan la información requerida para realizar el estudio.

\section{REFERENCIAS BIBLIOGRÁFICAS}

Adler, N. y Golany, B. (2001). Evaluation of Deregulated Airline Networks Using Data Envelopment Analysis Combined with Principal Component Analysis with an Application to Western Europe. European Journal of Operational Research, 132 (2), 260-273.

Ahmad, F.A., Ammar, A.G., Salamiah, A.J., y Khairun, N.A. (2015). Sustainable Tourism Development: A Study on Community Resilience for Rural Tourism in Malaysia, Procedia - Social and Behavioral Sciences, 168, 116-122.

Almeida, F., Pelaez, M. A., Balbuena, A., y Cortes, R. (2016). Residents' perceptions of tourism development in Benalmadena (Spain). Tourism Management, 54, 259-274.

Andereck, K. L., Valentine, K. M., Knopf, R. C., y Vogt, C. A. (2005). Residents' perceptions of community tourism impacts. Annals of Tourism Research, 32(4), 1056-1076.

Andereck, K. L., y Nyaupane, G. P. (2011). Exploring the nature of tourism and quality of life perceptions among residents. Journal of Travel Research, 50(3), 248-260.

Andersen, P. y Petersen, N.C. (1993). A procedure for ranking efficient units in Data Envelopment Analysis. Management Science, 39(10), 1261-1264.

Assaf A.G., Barros C.P. y Josiassen A. (2012). Hotel efficiency: A bootstrapped metafrontier approach. International Journal of Hospitality Management, 31, 621-629.

Barbot, C., Costa, A. y Sochirca, E. (2008). Airlines Performance in the New Market Context: A Comparative Productivity and Efficiency Analysis. Journal of Air Transport Management, 14 (5), 270-274.

Barros, C.P. y Dieke, P.U.C. (2007). Analyzing the total productivity change in travel agencies. Tourism Analysis, 12, 27-37.

Barros, C. P., y Mascarenhas, M. J. (2005). Technical and allocative efficiency in a chain of small hotels. International Journal of Hospitality Management, 24(3), 415-436.

Barros, C. P., Botti, L., Peypoch, N., Robinot, E., Solonandrasana, B. y Assaf, A. G. (2011). Performance of French destinations: Tourism attraction perspectives. Tourism Management, 32(1), 141-146.

Barros, C., Botti, L., Peypoch, N., y Solonandrasana, B. (2011). Managerial efficiency and hospitality industry: The Portuguese case. Applied Economics, 43, 2895-2905.

Beech, T., Dowd, M., Field, C., Hatcher, B. y Andréfouet, S. (2008). A Stochastic Approach to Marine Reserve Design: Incorporating Data Uncertainty. Ecological Informatics, 3 (4-5), 321-333. 
Bestard, A.B., y Nadal, R.J. (2007) Attitudes toward tourism and tourism congestion. Region et Developpement, 25, 193-207.

Blanke, J. y Chiesa, T. (2008). The Travel \& Tourism Competitiveness Index 2008: Measuring Key Elements Driving the Sector's Development. In The Travel \& Tourism Competitiveness Report 2008: Balancing Economic Development and Environmental Sustainability, 3-26. Geneva, Switzerland: World Economic Forum.

Boned, R., Monedero, C., De Juan, J.M., y Torres, J.C. (2006). Manual de Turismo sostenible: Araucaria XXI. Fundamentos y buenas prácticas. Agencia Española de Cooperación Internacional, Madrid.

Bosetti, V. y Locatelli, G. (2006). A Data Envelopment Analysis Approach to the Assessment of Natural Parks' Economic Efficiency and Sustainability. The Case of Italian National Parks. Sustainable Development, 14 (4), 277-286.

Bosetti, V., Cassinelli, M. y Lanza, A. (2003). Using data envelopment analysis to evaluate environmentally conscious tourism management. Paper prepared for the conference Tourism and Sustainable Development, Chia, Sardegna. Recuperado de http://citeseerx.ist.psu.edu/viewdoc/download?doi=10.1.1.563.4347\&rep=rep1\&type=pdf.

Bosetti, V., Cassinelli, M. y Lanza, A. (2006). Benchmarking in Tourism Destination, Keeping in Mind the Sustainable Paradigm. NRB - Natural Resources Management working paper. Recuperado de https://ageconsearch.umn.edu/bitstream/12156/1/wp060012.pdf.

Brida, J.G., Disegna, M., y Osti, L. (1999). Residents' perceptions of tourism impacts and attitudes towards tourism policies in a small mountain community. In Proceedings of the Ninth Canadian Congress on Leisure Research, Wolfville, NS, Canada, 12-15.

Busby, G., y Rendle, S. (2000). The transition from tourism on farms to farm tourism. Tourism Management, 21, 635-642.

Butler, R.W. (1999). Sustainable tourism: A state-of-the-art review. Tourism Geographies, 1(1), 7-25.

Butler, R. W. (2011). Sustainable tourism and the changing rural scene in Europe. In D. V. L. Macleod \& S. A. Gillespie (Eds.), Sustainable tourism in rural Europe (pp. 15-27). London: Routledge.

Cánoves, G., Villarino, M., Priestley, G. K., y Blanco, A. (2004). Rural tourism in Spain: an analysis of recent evolution. Geoforum, 35, 755-769.

Ceballos-Lascurain, H. (1996). Tourism, ecotourism and protected areas. Gland, Switzerland: IUCN, World Conservation Union.

Charnes A.W., Cooper, W.W., y Rhodes, E. (1978). Measuring the efficiency of decision making units. European Journal of Operational Research, 2, 429-444.

Chiang, W.E. (2006). A hotel performance evaluation of Taipei international tourist hotels using data envelopment analysis. Asia-Pacific Journal of Tourism Research, 11(1),29-42.

Copus, A.K., y Hörnström, L. (Eds) (2011). The new rural Europe: towards rural cohesion policy. Nordregio Report 2011:1. Nordregio, Stockholm.

Corraliza, J.A., Valero, E., y García, J. (2002): Los parques naturales en España: conservación y disfrute. Madrid: Fundación Alonso Martín Escudero.

Countryside Comission (1995). Sustainable rural tourism. Northampton (England). Departament of National Heritage. 
Cvetkosa, V. \& Barišić, P. (2017). The efficiency of the tourism industry in the Balkans. Proceedings of the Faculty of Economics in East Sarajevo, 14, 31-41.

Dearden, P., Bennett, M., y Johnston, J. (2003). Trends in global protected area governance, 1992-2002. Victoria, University of Victoria.

De Aranzábal, I., Schmitz, M.F., y Pineda, F.D. (2009). Integrating landscape analysis and planning: A multi-scale approach for oriented management of tourist recreation. Enviromental Management, 44 (5), 938-951. DOI: 10.1007/s00267-009-9371-z

De la Torre, G.M.V., Gutiérrez, E.M.A., y Guzman, T.J.L.G. (2005). Tourism as generator of wealth in rural areas. In Proceedings of the 18th European Advanced Studies Institute in Regional Science, Lodz-Cracow, Poland, 1-10.

Deery, M., Jagb, L., y Fredline, L. (2012). Rethinking social impacts of tourism research: a new research agenda. Tourism Management, 33 (1), 64-73. http://dx.doi.org/10.1016/j. tourman.2011.01.026

Deng, Y., Veiga, H., \& Wiper, M. P. (2019). Efficiency evaluation of hotel chains: a Spanish case study. SERIEs, 10(2), 115-139.

Eagles, P.F., McCool, S.F., y Haynes, C.D. (2002). Sustainable tourism in protected areas: Guidelines for planning and management. Switzerland and Cambridge, UICN Gland.

Eagles, P.F.J. y McCool, S.F. (2002). Tourism in National Parks and Protected Areas: Planning and Management. Wallingford: CABI.

Egbali, N., Nosrat, A.B., y Alipour, S.K.S. (2011). Effects of positive and negative rural tourism (Case study: Rural Semnan Province). Journal of Geography and Regional Planning, 4(2), 63-76.

Epler, M. (2002). Ecotourism: principles, practices \& policies for sustainability. Paris, United Nations Environment Programme (UNEP).

Eraqi, M.I. (2007). Local communities' attitudes towards impacts of tourism development in Egypt. Tourism Analysis, 12(3), 191-200.

Escudero, G. (1986). Actividad, ocupación y productividad agraria en España: un análisis de la población y del empleo. Revista de Estudios Agrosociales, 137, 379-417. Recuperado de http://www.mapama.gob.es/ministerio/pags/biblioteca/revistas/pdf reas/r137 29. $\underline{\mathrm{pdf}}$

Eshliki, S.A., Kaboudi, M. (2017). Perception of community in tourism impacts and their participation in tourism planning: Ramsar, Iran. Journal of Asian Behavioural Studies, 2(4), 59-69.

Europarc (2009). EUROPARC-España. Anuario 2009 del estado de los espacios naturales protegidos. Fundación Interuniversitaria Fernando González Bernáldez para los espacios naturales. Madrid.

Europarc (2018). EUROPARC-España. Anuario 2018 del estado de las áreas protegidas en España. Fundación Interuniversitaria Fernando González Bernáldez para los espacios naturales. Madrid.

Fennell, D. A. (2007). Ecotourism. New York: Routledge. Findlay AM, Short D \& Stockdale A 2000. The labour-market impact of migration to rural areas. Applied Geography, 20(4), 333-348. http://dx.doi.org/10.1016/S0143-6228(00)00012-6.

Finlay, A. M., Short,D.,yStockdale,A.(2000). The labour-market impact of migration to rural areas. Applied Geography, 20 (4), 333-348, https://doi.org/10.1016/S0143-6228(00)00012-6 
Fried, H. O., Lovell, C. A. K. y Schmidt, S. (2008). The Measurement of Productive Efficiency and Productivity Growth. Oxford: Oxford University Press.

Fuentes, R. (2011). Efficiency of travel agencies: A case study of Alicante, Spain. Tourism Management,32(1), 75-87.

Ghaderi, Z., y Henderson, J.C. (2012). Sustainable rural tourism in Iran: a perspective from Hawraman village. Tourism Management Perspectives, 2-3, 47-54. http://dx.doi. org/10.1016/i.tmp.2012.03.001

Garau, C. (2015). Perspectives on cultural and sustainable rural tourism in a smart region: The case study of Marmilla in Sardinia (Italy). Sustainability, 7, 6412-6434.

García, J.R., y Díaz, M.M. (2014). Planificación turística y desarrollo sostenible. Septem Ediciones.

Giannakis, E. (2014). The role of rural tourism on the development of rural areas: The case of Cyprus Elias. Romanian Journal of Regional Science, 8(1), 38-53.

Gómez-Limón, J., Múgica, M., y De Lucio, J.V. (2000). De la declaración a la gestión activa. Los espacios naturales protegidos del estado español en el umbral del siglo XXI, Fundación Fernando González Bernáldez, Madrid.

Green, H., C. Hunter, y B. Moore. (1990). Assessing the Environmental Impact of Tourism Development 1: Use of the Delphi Technique. Tourism Management, 11 (2), 111-120.

Hadad, S., Hadad, Y., Malul, M. y Rosenboim, M. (2012). The economic efficiency of the tourism industry: A global comparison. Tourism economics, 18, 5, 931-940.

Hair, J.F., Anderson, R.E., Tatham, R.L., y Black, W.C. (2000). Análisis Multivariante, Prentice Hall Iberia, Madrid.

Halseth, G., Markey, S., y Bruce, D. (2010). The next rural economies: constructing rural place in global economies. CABI Publishing, Wallingford.

Hardy, A.L., y Beeton, R.J.S. (2001). Sustainable Tourism or Maintainable Tourism: Managing Resources for More than Average Outcomes. Journal of Sustainable Tourism, 9 (3), 168-192.

Hill, B. (2005). The new rural economy: change, dynamism and government policy. The Institute of Economic Affairs, London.

Hockings, M., Stolton, S., Leverington, F., Dudley, N. y Courrau, J. (2006). Evaluating Effectiveness: A Framework for Assessing Management Effectiveness of Protected Areas. Second Edition. Gland, Switzerland and Cambridge, UK: IUCN.

Hof, J., Flather, C., Baltic, T. y King, R. (2004). Forest and Rangeland Ecosystem Condition Indicators: Identifying National Areas of Opportunity Using Data Envelopment Analysis. Forest Science, 50 (4), 473-494.

Huang, Y., Mesak, H. I., Hsu, M. K., \& Qu, H. (2012). Dynamic efficiency assessment of the Chinese hotel industry. Journal of Business Research, 65, 59-67.

Huh, C., y Vogt, C.A. (2008). Changes in residents' attitudes toward tourism over time: A cohort analytical approach. Journal of Travel Research, 46(4), 446-455.

Huybers, T. y Bennett, J. (2003). Environmental Management and the Competitiveness of Nature-Based Tourism Destinations. Environmental and Resource Economics, 24 (3), 213-233.

Hwang S.N., Chang T.Y. (2003). Using data envelopment analysis to measure hotel managerial efficiency change in Taiwan. Tourism Management, 24(4), 357-369. 
Jiménez, F.J. (2000). Turismo rural y empleo. VII Congreso de Economía Regional de Castilla y León. Soria.

Kaiser, H.F. (1960). The Application of Electronic Computers to Factor Analysis, Educational and Psychological Measurement, 10, 141-151.

Ko, D.W., y Stewart, W.P. (2002). A structural equation model of residents' attitudes for tourism development. Tourism Management, 23, 521-530.

Köksala, C.D. y Aksub, A. (2007). Efficiency evaluation of a-group travel agencies with data envelopment analysis (DEA): A case study in the Antalya region, Turkey. Tourism Management, 28(3), 830-834.

Ling-FengHsieh y Li-HungLin. (2010). A performance evaluation model for international tourist hotels in Taiwan-An application of the relational network DEA. International Journal of Hospitality Management, 29(1), 14-24.

Loncar, J. (2005). Globalization concept, origin and development trends. Geoadria, 10 (1), 91-104.

Long, P.H. (2011). Perceptions of tourism impact and tourism development among residents of Cuc Phuong National Park, Ninh Binh, Vietnam. J. Ritsumeikan. Social Sciences and Humanities, 3, 75-92.

Lozano, S. y Gutierrez, E. (2011). Efficiency analysis of EU-25 member states as tourist destinations. International Journal of Services Technology and Management, 15 (1/2), 69-88.

Lundmark, L. (2006). Restructuring and employment change in sparsely populated areas: examples from northern Sweden and Finland. Doctoral dissertation. Department of Social and Economic Geography, Umeå University, Umeå, Sweden.

Lundmark, L., Ednarsson, M., y Karlsson, S. (2014). International migration, self-employment and restructuring through tourism in sparsely populated areas. Scandinavian Journal of Hospitality and Tourism, 14(4), 422-440. http://dx.doi.org/10.1080/15022250.2014 .967995 .

Manasakis, C., Apostolakis, A. y Datseris, G. (2013). Using data envelopment analysis to measure hotel efficiency in Crete. International Journal of Contemporary Hospitality Management, 25(4), 510-535.

Mansfeld, Y.,y Jonas, A. Evaluating the socio-cultural carrying capacity of rural tourism communities: A 'value stretch' approach. Tijdschrift voor Economische en Sociale Geografie, 97(5), 583-601.

Martin, J. M., Salinas, J. A., Rodríguez, J. A, y Jiménez, J. D. (2017). Assessment of the tourism's potential as a sustainable development instrument in terms of annual stability: Application to Spanish rural destinations in process of consolidation. Sustainability, 9(10), 1692.

Martin, J. M., Salinas, J. A., y Rodríguez, J. A. (2018). Comprehensive evaluation of the tourism seasonality using a synthetic DP2 indicator. Tourism Geographies, 1-22. https://doi.org $\angle 10.1080 / 14616688.2018 .1505943$.

Mathieson, A., y Wall, G. (1982). Tourism: Economic, physical and social impacts. Harlow: Addison Wesley Longman.

Master, D. (1998). Marine wildlife tourism: Developing a quality approach in the highlands and islands. Inverness, Scotland: Tourism and Environment Initiative and Scottish Natural Heritage. 
McCool, S.F. (2006). Managing for Visitor Experiences in Protected Areas: Promising Opportunities and Fundamental Challenges. Parks: The International Journal for Protected Areas Managers, 16 (2), 3-9.

McGehee, N.G., y Andereck, K.L. (2004). Factors predicting rural residents' support of tourism. Journal of Travel Research, 43, 131-140.

Middleton, V., y Hawkins, R. (1998). Sustainable tourism. A Marketing Perspective. Oxford. Butterworth-Heinemann.

Mihalic, T. (2016). Sustainable-responsible tourism discourse e towards 'responsustable' tourism sustainability. Journal of Cleaner Production, 111, 461-470.

Min, Z., Xiaoli, P., y Bihu, W. (2012). Research on residents' perceptions on tourism impacts and attitudes: A case study of Pingyao ancient city. In Proceedings of the 6th Conference of the International Forum on Urbanism (IFoU), Tourbanism, Barcelona, 25-27 January 2012; pp. 1-10.

Morrison, F.D. (1987). Multivariate Statistical Methods, McGraw-Hill, Book Co, New York.

Mulero, A. y Garzón, R. (2005). Espacios naturales protegidos y desarrollo rural en España: los Planes de Desarrollo Sostenible. 68, 315-30.

Müller, D.K. (2006). Amenity migration and tourism development in the Tärna mountains, Sweden. In Moss LAG (ed). Amenity migrants: seeking and sustaining mountains and their cultures, 245-248. CABI, Wallingford.

Muresan, I.C., Oroian, C.F., Harun, R., Arion, F.H., Porutiu, A., Chiciudean, G.O., Todea, A., y Lile, R. (2016). Local Residents' Attitude toward Sustainable Rural Tourism Development. Sustainability, 8, 100.

Nanic, A., Barisic, P. y Vukovic, D. (2016). The efficiency of state aid on tourism development and supply of domestic products. 8th International conference, An Enterprise Odyssey: Saving the Sinking Ship Through Human Capital. Proceedings. Galetic, L., Načinovic Braje, I., Jakovic B. (Ed.), Zagreb, Croatia, June 8-11, 665-672.

Naughton-Treves, L., Buck Holland, M. y Brandon, k. (2005). The Role of Protected Areas in Conserving Biodiversity and Sustaining Local Livelihoods. Annual Review of Environment and Resources, 30, 219-252.

OCDE, (2006). The new rural paradigm: policies and governance. OECD rural policy reviews. Organisation for Economic Co-Operation and Development, Paris.

Ohe, Y. y Peypoch, N. (2016). Efficiency analysis of Japanese Ryokans A window DEA approach. Tourism economics: the business and finance of tourism and recreation, 1261-1273.

OMT, (2004). http://www.world-tourism.org/frameset/frame sustainable.html

Paniagua, A. (2002). Urban-ruralmigration, tourismentrepreneursandrural restructuringin Spain. Tourism Geographies, 4(4), 349-371. http://dx.doi.org/10.1080/14616680210158128.

Oral, M. y Yolalan, R. (1990). An empirical study on measuring operating efficiency and profitability of bank branc. European Journal of Operational Research, 46(3), 282-294.

Panyik, E., Costa, C., y Ratz, T. (2011). Implementing integrated rural tourism: an event-based approach. Tourism Management, 32, 1352-1363.

Pérez, C., y Zizumbo, L. (2014). Turismo rural y comunalidad: impactos socioterritoriales en San Juan Atzingo, México. Cuadernos de Desarrollo Rural, 11(73), 17-38. Recuperado de http://revistas.javeriana.edu.co/index.php/desarrolloRural/article/view/6108/7689 
Perrigot, R., Cliquet, G. y Piot-Lepetit, I. (2009). Plural form chain and efficiency: Insights from the French hotel chains and the DEA methodology. European Management Journal, 27(4), 268-280.

Pestana C. (2005). Measuring efficiency in the hotel industry: an illustrative example. Annals of tourism research, 32(2), 456-477.

Pestana, C. y Mascarenhas, M.J. (2005). Technical and allocative efficiency in a chain of small hotels. International Journal of hospitality Management, 24 (3), 415-436.

Plaza, J.I., y Araque, E. (2000). El turismo rural como fuente de desarrollo sostenible: best practices. Revista de Economía y Finanzas de Castilla y León, 4, 77-100.

Puczkó, L., y Rátz, T. (2000). Tourist and resident perceptions of the physical impacts of tourism at Lake Balaton, Hungary: Issues for sustainable tourism management. Journal of Sustainable Tourism, 8, 458-478.

Ramseook-Munhurrun, P., y Naidoo, P. (2011). Residents' attitudes toward perceived tourism benefits. International Journal Management Market Research, 4, 45-56.

Roberts, L., y Hall, D. (2001). Rural tourism and recreation: Principles to practice. Wallingford, England: $\mathrm{CABI}$.

Sánchez, A.M., y Sánchez, F.J. (2018). Impacto del turismo rural sobre el empleo en España: una aproximación a escala provincial. Cuadernos de Desarrollo Rural, 15 (82), 1-19. https://www.doi.org/10.11144/Javeriana.cdr15-82.itre

Sánchez-Zamora, P., Gallardo-Cobos, R., y Ceña-Delgado, F. (2014). Rural areas face the economic crisis: Analyzing the determinants of successful territorial dynamics. Journal of Rural Studies, 35, 11-25.

Santos, E., Fernández, A., y Muñoz, O. (2016). La incorporación del paisaje a la planificación turística. Análisis de la estrategia de turismo sostenible de Andalucía. Cuadernos de turismo, 37, 175-202. https://doi.org/10.6018/turismo.37.256201

Scheraga, C.A. (2004). Operational Efficiency versus Financial Mobility in the Global Airline Industry: A Data Envelopment and Tobit Analysis. Transportation Research Part A: Policy and Practice, 38 (5),383-404.

Seiford, L. M. (1997). A bibliography for data envelopment analysis (1978-1996). Annals of Operations Research, 73, 393-438.

Sellers-Rubio, R., y Casado-Díaz, A. B. (2018). Analyzing hotel efficiency from a regional perspective: The role of environmental determinants. International Journal of Hospitality Management, 75, 75-85.

Semenick, A. y Sickles, R.C. (2000). Time Series Analysis of Deregulatory Dynamics and Technical Efficiency: The Case of the US Airline Industry. International Economic Review, 41 (1), 203-218.

Shariff, N.M., Abidin, A.Z. (2013). Community attitude towards tourism impacts: Developing a standard instrument in the Malaysian context. Journal of Social Science Research, 1, 386-396.

Sharpley, R. (2000). Tourism and sustainable development: Exploring the theoretical divide. Journal of sustainable tourism, 8, 1-19.

Sharpley, R. (2003). Rural tourism and sustainability-a critique. En Hall. D., Robert, L., y Mitchell, M. New directions in rural tourism. Aldershot (England), Ashgate, 3-235. 
Sharpley, R., y Roberts, L. (2004). Rural Tourism - 10 years on. International Journal of Tourism Research, 6(3), 119-124.

Schmied, D. (Ed.) (2005). Winning and losing: the changing geography of Europe's rural areas. Ashgate, Aldershot.

Shucksmith, M., Talbot, H., y Lee, R. (2011). Meta-narratives as heuristic generalisations of rural change. In Copus AK \& Hörnström L (eds). The new rural Europe: towards rural cohesion policy, 19-36. Nordregio Report 2011:1. Nordregio, Stockholm.

Sigala, M., Airey, D., Jones, P., y Lockwood, A. (2004). ICT paradox lost? A stepwise DEA methodology to evaluate technology investments in tourism settings. Journal of Travel Research, 43, 180-192.

Solana-Ibáñez, J., Caravaca-Garratón, M., y Para-González, L. (2016). Two-stage data envelopment analysis of Spanish regions: Efficiency determinants and stability analysis. Contemporary Economics, 10(3), 259-274.

Soysal-Kurt, H. (2017). Measuring tourism efficiency of european countries by using Data Envelopment Analysis. European Scientific Journal, 13, 10, 31-49.

Stetic, S. (2012). Specific features of rural tourism destinations management. Journal of Settlements and Spatial Planning, 1, 131-137.

Su, B. (2011). Rural tourism in China. Tourism Management, 32, 1438-1441. https://doi. org/10.1016/i.tourman.2010.12.005

Tavares, G. (2002). A bibliography of data envelopment analysis (1978-2001). Rutcor Res, 1-2.

Troitiño, M. A., García-Blanco, F. J., García, M., del Río, M. I., Carpio, J., de la Calle, M., y Abad, L. D. (2005). Los espacios protegidos en España: significación e incidencia socioterritorial. Boletín de la Asociación de Geógrafos Españoles, 39, 227-265. Recuperado de https:// bage.age-geografia.es/ojs/index.php/bage/article/view/505/476

Troitiño, M.A. (1995). Espacios naturales protegidos y desarrollo rural: una relación territorial conflictiva. Boletín de la A.G.E, 20, 23-37.

Turek, F. (1999). Globalization and global security. Croatian Association for International Studies. Varazdin, 159.

UICN, (1994). Parques para la vida: Plan de Acción para las áreas protegidas de Europa. ICONA, Madrid.

Valdivieso, J.C., Eagles, P.F.J. y Gil, J.C. (2015). Efficient management capacity evaluation of tourism in protected áreas. Journal of Environmental Planning and Management, 58(9), 1544-1561. https://doi.org/10.1080/09640568.2014.937479

Wearing, S., y Neil, J. (2009). Ecotourism: impacts, potentials and possibilities. Oxford: Elsevier. Williams, P.W. y Ponsford, I.F. (2009). Confronting Tourism's Environmental Paradox: Transitioning for Sustainable Tourism. Futures, 41 (6), 396-404.

Woodley, S., Bertzky, B., Crawhall, N., Dudley, N., Londoño, J.M., MacKinnon, K., Redford, K. y Sandwith, T. (2012). Meeting Aichi Target 11: What Does Success Look like for Protected Area Systems? PARKS, 18 (1), 23.

Woods, M. (2011). Rural. Routledge, London.

Woods, M. (2005). Rural geography: processes, responses and experiences in rural restructuring. SAGE, London. 
Yoon, Y., Gursoy, D., y Chen, J. S. (1999). An investigation of the relationship between tourism impacts and host communities' characteristics. Anatolia. International Journal of Tourism and Hospitality Research, 10(1), 29-44.

ZhiYi, H. (2015). An analysis of the efficiency characteristics of the travel agency industry of China based on a DEA-Malmquist model. Tourism Tribune. 30(5), 23-30.

\section{CONTRIBUCIONES DE LOS AUTORES.}

\section{Autor 1. Francisca J. Sánchez-Sánchez}

Concepción original del trabajo.

Análisis, adquisición e interpretación de los datos.

Redacción y revisión crítica del contenido.

Aprobación final de la versión a publicar.

\section{Autor 2. Ana M. Sánchez-Sánchez}

Análisis, adquisición e interpretación de los datos.

Redacción y revisión crítica del contenido. 